\title{
GAUSSIAN HEAT KERNEL UPPER BOUNDS VIA PHRAGMÉN-LINDELÖF THEOREM
}

\author{
THIERRY COULHON AND ADAM SIKORA
}

\begin{abstract}
We prove that in presence of $L^{2}$ Gaussian estimates, so-called Davies-Gaffney estimates, on-diagonal upper bounds imply precise off-diagonal Gaussian upper bounds for the kernels of analytic families of operators on metric measure spaces.
\end{abstract}

\section{Contents}

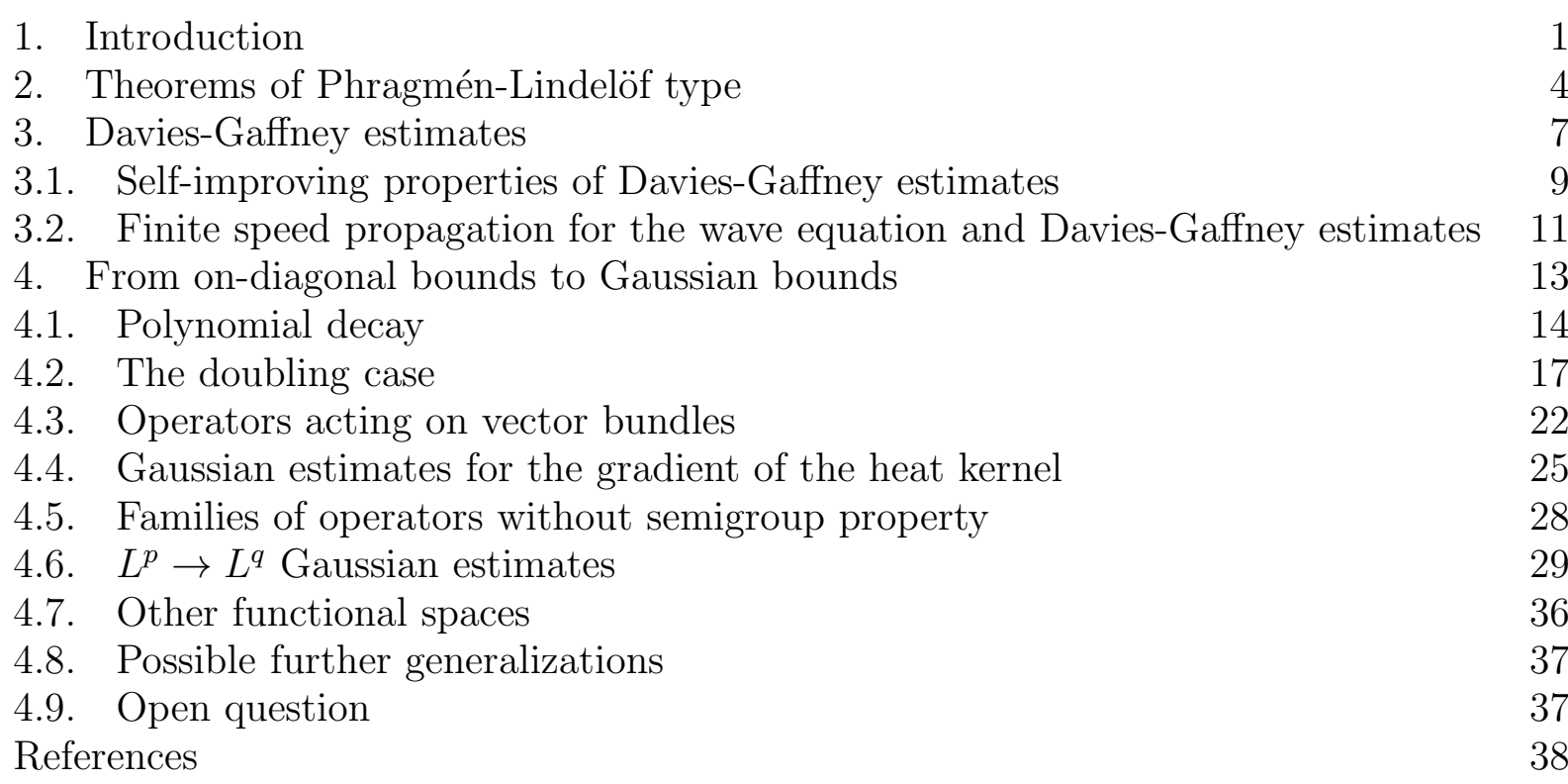

\section{INTRODUCTION}

The study of properties of second-order self-adjoint differential operators often depends on Gaussian upper bounds for the corresponding heat kernel, that is the kernel of the semigroup generated by these operators. Gaussian heat kernel upper bounds play a crucial role in the proofs of many results concerning boundedness of the Riesz transform, convergence of Bochner-Riesz means or boundedness of spectral multipliers, as well as problems related to

Date: September 4, 2018.

1991 Mathematics Subject Classification. 35K05, 58J35.

Key words and phrases. Heat kernels, Gaussian bounds, Phragmén-Lindelöf theorem.

TC's research was partially supported by the European Commission (IHP Network "Harmonic Analysis and Related Problems" 2002-2006, Contract HPRN-CT-2001-00273-HARP).

AS's research was partially supported by an Australian Research Council (ARC) Discovery Grant DP 0451016 and New Mexico State University Summer Research Award. 
maximal regularity properties (see for example the articles 18, 20, 21, 43, 4, 37, 15, 1, 2, 14, 28, 19] and the monograph [46]). If $p_{t}(x, y)$ denotes the heat kernel corresponding to a second-order differential elliptic or sub-elliptic operator, then the typical Gaussian heat kernel upper bound is of the form

$$
0 \leq p_{t}(x, y) \leq \frac{C}{V(x, \sqrt{t})} \exp \left(-\frac{d^{2}(x, y)}{C t}\right)
$$

for all $t>0, x, y$ ranging in the space where the operator acts. For instance, if $p_{t}$ is the kernel corresponding to the Laplace-Beltrami operator on a Riemannian manifold, then, at least in some favorable cases, one expects $V(x, r)$ to be equal to the volume of the geodesic ball of radius $r$ and centered at $x$ and $d$ denotes the Riemannian distance. In the standard approach, proofs of Gaussian estimates are divided into two steps. First one obtains ondiagonal estimates

$$
p_{t}(x, x) \leq \frac{C}{V(x, \sqrt{t})}
$$

for all $t, x$. Then the theory says that one can automatically improve on-diagonal bounds by adding the Gaussian factor $\exp \left(-\frac{d^{2}(x, y)}{C t}\right)$ and obtain this way Gaussian bounds (1.1). There are basically three known methods to derive Gaussian bounds from on-diagonal bounds (1.2): Davies's perturbation method (see [23, [26], [16]), the integrated maximum principle (see 33], 34, 35]) and finite propagation speed for the wave equation (see [49, 51]).

The main aim of the present paper is to introduce a new method for deducing Gaussian bounds from uniform bounds (1.2), which relies mainly on the Phragmén-Lindelöf theorem. Our approach is closely related to the main idea behind complex interpolation, and it shows that surprisingly the Gaussian bounds and the complex interpolation results are of similar nature. This allows us to look at the off-diagonal Gaussian bounds from a new perspective. The use of Phragmén-Lindelöf theorems for heat kernel estimates was introduced to our knowledge in [25, see in particular Lemma 9, see also [23, Theorem 3.4.8, p.103]. In [25], Davies uses Gaussian bounds for real time and the Phragmén-Lindelöf technique to obtain complex time estimates for the heat kernel, that is estimates for $p_{z}(x, y)$ for all $z \in \mathbb{C}_{+}$, where $\mathbb{C}_{+}$is the complex half-plane $\operatorname{Re} z>0$. Roughly speaking, in our approach, we reverse the order of Davies's idea and we use the Phragmén-Lindelöf technique to obtain both real and complex time Gaussian bounds. Our method yields more precise complex time Gaussian bounds than in [25] and [23, Theorem 3.4.8, p.103].

We express the assumptions of our results in terms of so-called Davies-Gaffney estimates. To our knowledge, these estimates were formulated for the first time in [24], but according to Davies himself the idea stems from 31. Davies-Gaffney estimates hold for essentially all selfadjoint, elliptic or subelliptic second-order differential operators including Laplace-Beltrami operators on complete Riemannian manifolds, Schrödinger operators with real-valued potentials and electromagnetic fields, and Hodge-Laplace operators acting on differential forms (see Theorem 3.3 and Section 4.3 below). A discrete time version of the Davies-Gaffney estimate is discussed in [22 ${ }^{1}$. Davies-Gaffney estimates are also easy to obtain. For non-negative self-adjoint operators, they are equivalent with the finite speed propagation property for the

\footnotetext{
${ }^{1}$ However, it is not clear how to extend the methods of the present work to the discrete time case, in order to replace the use of the rather technical discrete integrated maximum principle as in [22].
} 
corresponding wave equation (see 51] and Section 3.2 below). We discuss this equivalence here as a simple but illuminating application of the Phragmén-Lindelöf technique.

Our approach allows us to obtain far reaching generalizations of the results obtained in [23, 26, 16, 33, 34, 35, 49, 51. In the present paper, we do not have to assume anything about the nature of the infinitesimal generator of the semigroup under consideration; in particular, the generating operator does not have to be a second-order differential operator, and the semigroup does not have to be Markov. Our method works also for operators acting on differential forms and more generally on vector bundles ${ }^{2}$. Actually, instead of considering the analytic semigroup $\left\{\exp (-z L): z \in \mathbb{C}_{+}\right\}$generated by some non-negative self-adjoint operator $L$, we are able to study any uniformly bounded analytic family of operators $\left\{\Psi(z): z \in \mathbb{C}_{+}\right\}$. We do not have to assume that $\Psi$ has the semigroup property nor that $\Psi(z)$ is a linear operator, as far as $\left\{\Psi(z): z \in \mathbb{C}_{+}\right\}$satisfies the Davies-Gaffney estimates (see (3.2) below). For example we can study the estimates for the gradient of the heat kernel in the same way as the estimates for the heat kernel itself. We are also able to consider the family given by the formula $\Psi(z)=\exp (-z L)-\exp \left(-z L^{0}\right)$ where $L, L^{0}$ are different generators of analytic semigroups. For example, one can consider the situation where $L$ is an operator with periodic coefficients in divergence form and $L^{0}$ is its homogenization, to obtain Gaussian estimates for the difference of the corresponding heat kernels $\left|p_{t}(x, y)-p_{t}^{0}(x, y)\right|$.

Next, our methods have various applications in the theory of $L^{p}$ to $L^{q}$ Gaussian estimates, developed by Blunck and Kunstmann in [8, 9, 10, 11, 12, see also [42] and [5]. Blunck and Kunstmann call such estimates generalized Gaussian estimates. They use generalized Gaussian estimates to study $L^{p}$ spectral multipliers for operators without heat kernels. Our approach provides a strong tool to verify the generalized Gaussian estimates for a large class of operators. It is natural here to consider not only $L^{p}$ spaces but other functional spaces. This leads to another generalization of Gaussian estimates (see Section 4.7 below).

Before we introduce all technical details needed to state our main results, we would like to discuss Theorems 1.1 and 1.2 below, which are only specific consequences of these results, but provide a good non-technical illustration of our approach. In [23, Theorem 3.4.8 p.103] (see also [25, Lemma 9]) Davies shows that the Gaussian estimate for the heat kernel extends to complex values of time. The surprisingly simple proof of Theorem 1.1 yields a more precise version of [23, Theorem 3.4 .8 p.103], and at the same time it provides an alternative proof of real time off-diagonal Gaussian bounds obtained in [23, 26, 16. Recall that the heat semigroup $\exp (-t \Delta)$ generated by the (non-negative) Laplace-Beltrami operator $\Delta$ on a complete Riemannian manifold $M$ is self-adjoint on $L^{2}(M)$ with a smooth positive kernel $p_{t}(x, y), t>0, x, y \in M$, called the heat kernel on $M$; it extends to a complex time semigroup $\exp (-z \Delta), z \in \mathbb{C}_{+}$, with a smooth kernel $p_{z}(x, y), z \in \mathbb{C}_{+}, x, y \in M$. Denote by $d$ the geodesic distance on $M$.

Theorem 1.1. Let $p_{z}, z \in \mathbb{C}_{+}$, be the heat kernel on a complete Riemannian manifold $M$. Suppose that

$$
p_{t}(x, x) \leq K t^{-D / 2}, \forall t>0, x \in M
$$

\footnotetext{
${ }^{2}$ It is worth noting at this point that there is a connection between estimates of the heat kernel on 1-forms and the $L^{p}$ boundedness of the Riesz transform for $p>2$ (see for example [21, 20, 51]).
} 
for some $K$ and $D>0$. Then

$$
\left|p_{z}(x, y)\right| \leq e K(\operatorname{Re} z)^{-D / 2}\left(1+\operatorname{Re} \frac{d^{2}(x, y)}{4 z}\right)^{D / 2} \exp \left(-\operatorname{Re} \frac{d^{2}(x, y)}{4 z}\right)
$$

for all $z \in \mathbb{C}_{+}, x, y \in M$.

For $z=t \in \mathbb{R}_{+}$, estimates (1.4) can still be improved. It is possible to prove that

$$
0 \leq p_{t}(x, y) \leq C t^{-D / 2}\left(1+\frac{d^{2}(x, y)}{4 t}\right)^{(D-1) / 2} \exp \left(-\frac{d^{2}(x, y)}{4 t}\right)
$$

(see [49]). Moreover, it is known that the additional term $\left(1+\frac{d^{2}(x, y)}{4 t}\right)^{(D-1) / 2}$ cannot be removed in general from (1.5). See [45] for a counterexample. However, using the PhragménLindelöf technique we obtain the following variation of Theorem [1.1.

Theorem 1.2. Let $p_{z}, z \in \mathbb{C}_{+}$, be the heat kernel on a complete Riemannian manifold $M$. Suppose that

$$
\left|p_{z}(x, y)\right| \leq K|z|^{-D / 2}, \forall z \in \mathbb{C}_{+}, x, y \in M,
$$

for some Kand $D>0$. Then

$$
\left|p_{z}(x, y)\right| \leq e K|z|^{-D / 2} \exp \left(-\operatorname{Re} \frac{d^{2}(x, y)}{4 z}\right)
$$

for all $z \in \mathbb{C}_{+}, x, y \in M$.

Theorems 1.1 and 1.2 are straightforward consequences of Theorems 4.1 and 4.2 below, and the well-known fact that the Laplace-Beltrami operator on complete Riemannian manifolds satisfies Davies-Gaffney estimates, see the remark after Theorem [3.3 below or [24, 35].

Theorem 1.2 shows that one can remove the additional factor $\left(1+\frac{d^{2}(x, y)}{t}\right)^{D / 2}$ in (1.5) if one is able to replace estimates (1.3) by the stronger ones (1.6). This is an example of a result which we can obtain using Phragmén-Lindelöf technique and which does not seem to follow from the techniques developed in [23, 26, 16, 33, 34, 35, 49, 51].

\section{Theorems of Phragmén-Lindelöf type}

Let us start with stating the Phragmén-Lindelöf theorem for sectors.

Theorem 2.1. Let $S$ be the open region in $\mathbb{C}$ bounded by two rays meeting at an angle $\pi / \alpha$, for some $\alpha>1 / 2$. Suppose that $F$ is analytic on $S$, continuous on $\bar{S}$, and satisfies $|F(z)| \leq C \exp \left(c|z|^{\beta}\right)$ for some $\beta \in[0, \alpha)$ and for all $z \in S$. Then the condition $|F(z)| \leq B$ on the two bounding rays implies $|F(z)| \leq B$ for all $z \in S$.

For the proof see [44, Theorem 7.5, p.214, vol.II] or [52, Lemma 4.2, p.108]. Propositions 2.2, 2.3 and 2.4 are simple consequences of Theorem 2.1.

Proposition 2.2. Suppose that $F$ is an analytic function on $\mathbb{C}_{+}$. Assume that, for given numbers $A, B, \gamma>0, a \geq 0$,

$$
\begin{gathered}
|F(z)| \leq B, \quad \forall z \in \mathbb{C}_{+}, \quad \text { and } \\
|F(t)| \leq A e^{a t} e^{-\frac{\gamma}{t}}, \quad \forall t \in \mathbb{R}_{+} .
\end{gathered}
$$


Then

$$
|F(z)| \leq B \exp \left(-\operatorname{Re} \frac{\gamma}{z}\right), \quad \forall z \in \mathbb{C}_{+} .
$$

Proof. Consider the function

$$
u(\zeta)=F\left(\frac{\gamma}{\zeta}\right)
$$

which is also defined on $\mathbb{C}_{+}$. By (2.1),

$$
\left|u(\zeta) e^{\zeta}\right| \leq B \exp |\zeta|, \quad \forall \zeta \in \mathbb{C}_{+}
$$

Again by (2.1) we have, for any $\varepsilon>0$,

$$
\sup _{\operatorname{Re} \zeta=\varepsilon}\left|u(\zeta) e^{\zeta}\right| \leq B e^{\varepsilon}
$$

By (2.2),

$$
\sup _{\zeta \in[\varepsilon, \infty)}\left|u(\zeta) e^{\zeta}\right| \leq A e^{a \gamma / \varepsilon}
$$

Hence, by Phragmén-Lindelöf theorem with angle $\pi / 2$ and $\beta=1$, applied to

$$
S_{\varepsilon}^{+}=\{z \in \mathbb{C}: \operatorname{Re} z>\varepsilon \text { and } \operatorname{Im} z>0\}
$$

and

$$
S_{\varepsilon}^{-}=\{z \in \mathbb{C}: \operatorname{Re} z>\varepsilon \text { and } \operatorname{Im} z<0\}
$$

one obtains

$$
\sup _{\operatorname{Re} \zeta \geq \varepsilon}\left|u(\zeta) e^{\zeta}\right| \leq \max \left\{A e^{a \gamma / \varepsilon}, B e^{\varepsilon}\right\}, \quad \forall \varepsilon>0 .
$$

Now by the Phragmén-Lindelöf theorem with angle $\pi$ and $\beta=0$,

$$
\sup _{\operatorname{Re} \zeta \geq \varepsilon}\left|u(\zeta) e^{\zeta}\right| \leq B e^{\varepsilon}, \quad \forall \varepsilon>0
$$

Letting $\varepsilon \rightarrow 0$ we obtain

$$
\sup _{\operatorname{Re} \zeta>0}\left|u(\zeta) e^{\zeta}\right| \leq B
$$

This proves (2.3) by putting $\zeta=\frac{\gamma}{z}$.

Note that the estimate (2.3) does not depend on constants $A, a$ in (2.2). This simple observation is the heart of the matter in the present paper.

The above proposition will be used to prove the equivalence between the finite speed propagation property for the solution of the wave equation and Davies-Gaffney estimates (see $\$ 3.2$ below). However, in order to study the Gaussian bounds for heat kernels, we shall need a more sophisticated version of Proposition 2.2 .

Given $\gamma>0$, denote by $\mathcal{C}_{\gamma}$ the closed disk in $\mathbb{C}_{+}$centered on the real axis, tangent to the imaginary axis, with radius $\gamma / 2$, that is the region

$$
\mathcal{C}_{\gamma}=\left\{z \in \mathbb{C} \backslash\{0\}: \operatorname{Re} \frac{\gamma}{z} \geq 1\right\}
$$


Proposition 2.3. Let $F$ be an analytic function on $\mathbb{C}_{+}$. Assume that, for given numbers $A, B, \gamma, \nu>0$,

$$
|F(z)| \leq A
$$

for all $z \in \mathbb{C}_{+}$;

$$
|F(t)| \leq A e^{-\frac{\gamma}{t}}
$$

for all $t \in \mathbb{R}_{+}$such that $t \leq \gamma$;

$$
|F(z)| \leq B\left(\frac{\operatorname{Re} z}{4 \gamma}\right)^{-\nu / 2}
$$

for all $z \in \mathcal{C}_{\gamma}$. Then

$$
|F(z)| \leq e B\left(\frac{2 \gamma}{|z|}\right)^{\nu} \exp \left(-\operatorname{Re} \frac{\gamma}{z}\right)
$$

for all $z \in \mathcal{C}_{\gamma}$.

Proof. Consider again the function $u$ defined by (2.4). It satisfies condition (2.5) and (2.6) with $B=A, a=0$ and $\varepsilon=1$. Hence by (2.7)

$$
\sup _{\operatorname{Re} \zeta \geq 1}\left|u(\zeta) e^{\zeta}\right| \leq e A
$$

Consider now the function $v$ defined on $\mathbb{C}_{+}$by the formula

$$
v(\zeta)=(2 \zeta)^{-\nu} u(\zeta) e^{\zeta}
$$

Note that $|v(\zeta)| \leq 2^{-\nu}\left|u(\zeta) e^{\zeta}\right|$ for $\operatorname{Re} \zeta \geq 1$ so by (2.12) $v$ is bounded on the set $\operatorname{Re} \zeta \geq 1$. Now, by Phragmén-Lindelöf theorem with angle $\pi$ and $\beta=0$,

$$
\sup _{\operatorname{Re} \zeta \geq 1}|v(\zeta)|=\sup _{\operatorname{Re} \zeta=1}|v(\zeta)| \text {. }
$$

Put $\zeta=1+i s$. By (2.10),

$$
\begin{aligned}
\sup _{\operatorname{Re} \zeta=1}|v(\zeta)| & =\sup _{\operatorname{Re} \zeta=1}\left|(2 \zeta)^{-\nu} F\left(\frac{\gamma}{\zeta}\right) e^{\zeta}\right| \\
& \leq \sup _{\operatorname{Re} \zeta=1} e B|2 \zeta|^{-\nu}\left(\operatorname{Re} \frac{1}{4 \zeta}\right)^{-\nu / 2} \\
& \leq e B \sup _{s \in \mathbb{R}}\left(1+s^{2}\right)^{-\nu / 2}\left(\frac{1}{1+s^{2}}\right)^{-\nu / 2}=e B .
\end{aligned}
$$

Hence

$$
\sup _{\operatorname{Re} \zeta \geq 1}|v(\zeta)| \leq e B
$$

Now we put $\zeta=\frac{\gamma}{z}$ in (2.13) and we obtain

$$
\left|\frac{2 \gamma}{z}\right|^{-\nu}|F(z)| \exp \left(\operatorname{Re} \frac{\gamma}{z}\right) \leq e B
$$

This proves (2.11) for all $z \in \mathcal{C}_{\gamma}$. 
Finally let us discuss one more version of Proposition 2.3. We shall need this modified version to prove Theorem 1.2. The main difference between Propositions 2.3 and 2.4 is that we multiply the function $F$ by an analytic function $e^{g}$ satisfying a growth condition.

Proposition 2.4. Let $g$ and $F$ be analytic functions on $\mathbb{C}_{+}$. Assume that, for given numbers $C, c, \gamma>0$ and $0 \leq \beta<1$,

$$
|\exp g(z)| \leq C e^{c|z|^{-\beta}}
$$

for all $z \in \mathcal{C}_{\gamma}$.

Next assume that $F$ satisfies conditions (2.8) and (2.9) and that

$$
|F(z)| \leq B\left(\frac{\operatorname{Re} z}{4 \gamma}\right)^{-\nu / 2} \exp (-\operatorname{Re} g(z))
$$

for some $\nu>0$ and all $z \in \mathcal{C}_{\gamma}$. Then

$$
|F(z)| \leq e B\left(\frac{2 \gamma}{|z|}\right)^{\nu} \exp \left(-\operatorname{Re} g(z)-\operatorname{Re} \frac{\gamma}{z}\right)
$$

for all $z \in \mathcal{C}_{\gamma}$.

Proof. Define functions $u_{1}$ and $v_{1}$ on $\mathbb{C}_{+}$by the formulae

$$
v_{1}(\zeta)=(2 \zeta)^{-\nu} u_{1}(\zeta) e^{\zeta}=(2 \zeta)^{-\nu} u(\zeta) \exp \left(g\left(\frac{\gamma}{\zeta}\right)\right) e^{\zeta}=(2 \zeta)^{-\nu} F\left(\frac{\gamma}{\zeta}\right) \exp \left(g\left(\frac{\gamma}{\zeta}\right)\right) e^{\zeta}
$$

By (2.12) and (2.14),

$$
\left|v_{1}(\zeta)\right| \leq 2^{-\nu}\left|u_{1}(\zeta) e^{\zeta}\right|=2^{-\nu}\left|u(\zeta) e^{\zeta}\right|\left|\exp \left(g\left(\frac{\gamma}{\zeta}\right)\right)\right| \leq 2^{-\nu} e A C e^{c \gamma^{-\beta}|\zeta|^{\beta}}
$$

if $\operatorname{Re} \zeta \geq 1$. Now by Phragmén-Lindelöf theorem with angle $\pi$

$$
\sup _{\operatorname{Re} \zeta \geq 1}\left|v_{1}(\zeta)\right|=\sup _{\operatorname{Re} \zeta=1}\left|v_{1}(\zeta)\right|
$$

By (2.15),

$$
\sup _{\operatorname{Re} \zeta=1}\left|v_{1}(\zeta)\right| \leq \sup _{\operatorname{Re} \zeta=1} e B|2 \zeta|^{-\nu}\left(\operatorname{Re} \frac{1}{4 \zeta}\right)^{-\nu / 2}
$$

and the rest of the proof is as in Proposition 2.3 .

\section{Davies-Gaffney estimates}

Let $(M, d, \mu)$ be a metric measure space, that is $\mu$ is a Borel measure with respect to the topology defined by the metric $d$. Next let $B(x, r)=\{y \in M, d(x, y)<r\}$ be the open ball with center $x \in M$ and radius $r>0$. For $1 \leq p \leq+\infty$, we denote the norm of a function $f \in L^{p}(M, d \mu)$ by $\|f\|_{p}$, by $\langle.,$.$\rangle the scalar product in L^{2}(M, d \mu)$, and if $T$ is a bounded linear operator from $L^{p}(M, d \mu)$ to $L^{q}(M, d \mu), 1 \leq p, q \leq+\infty$, we write $\|T\|_{p \rightarrow q}$ for the operator norm of $T$.

Suppose that, for every $z \in \mathbb{C}_{+}, \Psi(z)$ is a bounded linear operator acting on $L^{2}(M, d \mu)$ and that $\Psi(z)$ is an analytic function of $z$. Assume in addition that

$$
\|\Psi(z)\|_{2 \rightarrow 2} \leq 1, \quad \forall z \in \mathbb{C}_{+} .
$$


For $U_{1}, U_{2} \subset M$ open subsets of $M$, let $d\left(U_{1}, U_{2}\right)=\inf _{x \in U_{1}, y \in U_{2}} d(x, y)$. We say that the family $\left\{\Psi(z): z \in \mathbb{C}_{+}\right\}$satisfies the Davies-Gaffney estimate if

$$
\left|\left\langle\Psi(t) f_{1}, f_{2}\right\rangle\right| \leq \exp \left(-\frac{r^{2}}{4 t}\right)\left\|f_{1}\right\|_{2}\left\|f_{2}\right\|_{2}
$$

for all $t>0, U_{i} \subset M, f_{i} \in L^{2}\left(U_{i}, d \mu\right), i=1,2$ and $r=d\left(U_{1}, U_{2}\right)$. Note that we only assume that (3.2) holds for positive real $t$.

A slightly different form of Davies-Gaffney estimate is mostly considered in the literature (see for instance [24] or [35]): in our notation, it reads

$$
\left|\left\langle\Psi(t) \chi_{U_{1}}, \chi_{U_{2}}\right\rangle\right| \leq \exp \left(-\frac{r^{2}}{4 t}\right) \sqrt{\mu\left(U_{1}\right) \mu\left(U_{2}\right)}
$$

where $\chi_{U}$ denotes the characteristic function of the set $U$. Of course, (3.3) follows from (3.2) by taking $f_{1}=\chi_{U_{1}}$ and $f_{2}=\chi_{U_{2}}$. Conversely, assume (3.3) and let $f_{i}=\sum_{j} c_{j}^{i} \chi_{A_{j}^{i}}$, where $A_{j}^{i} \subset U_{i}$. Then

$$
\begin{aligned}
<\Psi(t) f_{1}, f_{2}> & \leq \sum_{j} \sum_{\ell}\left|c_{j}^{1} c_{\ell}^{2}\right|\left(\mu\left(A_{j}^{1}\right) \mu\left(A_{\ell}^{2}\right)\right)^{1 / 2} \exp \left(-\frac{d^{2}\left(A_{j}^{1}, A_{\ell}^{2}\right)}{4 t}\right) \\
& \leq \sum_{j} \sum_{\ell}\left|c_{j}^{1} c_{l}^{2}\right|\left(\mu\left(A_{j}^{1}\right) \mu\left(A_{\ell}^{2}\right)\right)^{1 / 2} \exp \left(-\frac{d^{2}\left(U_{1}, U_{2}\right)}{4 t}\right) .
\end{aligned}
$$

By (3.1), $<\Psi(z) f_{1}, f_{2}>\leq\left\|f_{1}\right\|_{2}\left\|f_{2}\right\|_{2}$. Proposition 2.2 then yields (3.2) for such $f_{1}, f_{2}$, and one concludes by density.

One may wonder what is the justification of the constant 4 in (3.2); we shall see in Theorem 3.4 below that in the case where $\Psi(z)$ is a semigroup $e^{-z L}, 4$ is the good normalisation between the operator $L$ and the distance $d$, namely it translates the fact that the associated wave equation has propagation speed 1.

The other constants in (3.1) and (3.2) have been normalized to one for simplicity, anyway then can be absorbed by multiplying accordingly the family $\Psi(z)$.

3.0.1. Examples. Semigroups of operators generated by non-negative self-adjoint operators always satisfy (3.1), and among them many examples of interest satisfy (3.2). Recall that, if $L$ is a non-negative self-adjoint operator on $L^{2}(M, d \mu)$, one can construct the spectral decomposition $E_{L}(\lambda)$ of the operator $L$. For any bounded Borel function $m:[0, \infty) \rightarrow \mathbb{C}$, one then defines the operator $m(L): L^{2}(M, d \mu) \rightarrow L^{2}(M, d \mu)$ by the formula

$$
m(L)=\int_{0}^{\infty} m(\lambda) \mathrm{d} E_{L}(\lambda)
$$

Now, for $z \in \mathbb{C}_{+}$and $m_{z}(\lambda)=\exp (-z \lambda)$, one sets $m_{z}(L)=\exp (-z L), z \in \mathbb{C}_{+}$. By spectral theory the family $\Psi(z)=\left\{\exp (-z L): z \in \mathbb{C}_{+}\right\}$, also called semigroup of operators generated by $L$, satisfies condition (3.1).

As we already said, condition (3.2) holds for all kinds of self-adjoint, elliptic, second order like operators. Condition (3.2) is well-known to hold for Laplace-Beltrami operators on all complete Riemannian manifolds. More precisely, Condition (3.3) is proved for such operators in [24] and 35]. See also the remark after Theorem 3.3. In the more general setting of Laplace type operators acting on vector bundles, condition (3.2) is proved in [51]. Another important class of semigroups satisfying condition (3.2) are semigroups generated 
by Schrödinger operators with real potential and magnetic field (see for example [50], as well as Theorem 3.3 and Section 4.3 below).

Note that self-adjointness and non-negativity of $L$ are a way to ensure that $\exp (-z L)$ is defined for $z \in \mathbb{C}_{+}$, and (3.1), but these conditions may hold for non-self-adjoint $L$, and they are sufficient by themselves to run the rest of our theory.

Estimates (3.2) also hold in the setting of local Dirichlet forms (see for example 38, Theorem 2.8], and also [53, 54]). In this case the metric measure spaces under consideration are possibly not equipped with any differential structure. However, the semigroups associated with these Dirichlet forms do satisfy in general Davies-Gaffney estimates with respect to an intrinsic distance.

In the sequel, if $(M, d, \mu)$ is a metric measure space and $L$ a non-negative self-adjoint operator on $L^{2}(M, d \mu)$, we shall say abusively that $(M, d, \mu, L)$ satisfies the Davies-Gaffney condition if (3.2) holds with $\Psi(t)=e^{-t L}$.

3.1. Self-improving properties of Davies-Gaffney estimates. It is convenient to establish two simple lemmas concerning Davies-Gaffney estimates before discussing our main results. First we observe that given (3.1) it is enough to test (3.2) on balls only. Then we observe that any additional multiplicative constant or even additional exponential factor in (3.2) can be replaced by the constant in (3.1).

Lemma 3.1. Suppose that $(M, d, \mu)$ is a separable metric space and that the analytic family $\left\{\Psi(z): z \in \mathbb{C}_{+}\right\}$of bounded operators on $L^{2}(M, d \mu)$ satisfies condition (3.1), and condition (3.2) restricted to all balls $U_{i}=B\left(x_{i}, r_{i}\right), i=1,2$, for all $x_{1}, x_{2} \in M, r_{1}, r_{2}>0$. Then it satisfies condition (3.2) for all open subsets $U_{1}, U_{2}$.

Proof. Let $U_{1}$ and $U_{2}$ be arbitrary open subsets of $M$; set $r=d\left(U_{1}, U_{2}\right)$. Let $f=\sum_{i=1}^{k} f_{i}$, where for all $1 \leq i \leq k, f_{i} \in L^{2}\left(B\left(x_{i}, r_{i}\right), d \mu\right), B\left(x_{i}, r_{i}\right) \subset U_{1}$, and $f_{i_{1}}(x) f_{i_{2}}(x)=0$ for all $x \in M, 1 \leq i_{1}<i_{2} \leq k$. Similarly let $g=\sum_{j=1}^{\ell} g_{j}$ where $g_{j} \in L^{2}\left(B\left(y_{j}, s_{j}\right), d \mu\right)$, $B\left(y_{j}, s_{j}\right) \subset U_{2}$ for all $1 \leq j \leq \ell$, and $g_{j_{1}}(x) g_{j_{2}}(x)=0$ for all $x \in M, 1 \leq j_{1}<j_{2} \leq \ell$. Note that $d\left(B\left(x_{i}, r_{i}\right), B\left(y_{j}, s_{j}\right)\right) \geq r$. Now if condition (3.2) holds for balls then

$$
\begin{aligned}
|\langle\Psi(t) f, g\rangle| & =\left|\left\langle\Psi(t) \sum_{i=1}^{k} f_{i}, \sum_{j=1}^{\ell} g_{j}\right\rangle\right| \\
& =\sum_{i=1}^{k} \sum_{j=1}^{\ell}\left|\left\langle\Psi(t) f_{i}, g_{j}\right\rangle\right| \\
& \leq \sum_{i=1}^{k} \sum_{j=1}^{\ell} e^{-\frac{r^{2}}{4 t}}\left\|f_{i}\right\|_{2}\left\|g_{j}\right\|_{2} \\
& \leq e^{-\frac{r^{2}}{4 t}}\left(\sum_{i=1}^{k}\left\|f_{i}\right\|_{2}\right)\left(\sum_{j=1}^{\ell}\left\|g_{j}\right\|_{2}\right) \\
& \leq e^{-\frac{r^{2}}{4 t}} \sqrt{k \ell}\left(\sum_{i=1}^{k}\left\|f_{i}\right\|_{2}^{2}\right)^{1 / 2}\left(\sum_{j=1}^{\ell}\left\|g_{j}\right\|_{2}^{2}\right)^{1 / 2} \\
& =e^{-\frac{r^{2}}{4 t}} \sqrt{k \ell}\|f\|_{2}\|g\|_{2} .
\end{aligned}
$$


Now we assume that (3.1) holds so if we put $F(z)=\langle\Psi(z) f, g\rangle$ then Proposition 2.2 shows that the term $C k l$ in the above inequality can be replaced by 1 . This means that (3.2) holds for $f$ and $g$. Now to finish the proof of the lemma, it is enough to note that, since $M$ is separable, the space of all possible finite linear combinations of functions $f$ such that $\operatorname{supp} f \subset B(x, r) \subset U$ is dense in $L^{2}(U, d \mu)$. Moreover, if $f=\sum_{i=1}^{k} f_{i}$ and $f_{i} \in$ $L^{2}\left(B\left(x_{i}, r_{i}\right), d \mu\right)$ for all $1 \leq i \leq k$ then there exist functions $\tilde{f}_{i} \in L^{2}\left(B\left(x_{i}, r_{i}\right), d \mu\right)$ such that $f=\sum_{i=1}^{k} \tilde{f}_{i}$ and in addition, for all $1 \leq i_{1}<i_{2} \leq k, \tilde{f}_{i_{1}}(x) \tilde{f}_{i_{2}}(x)=0$ for all $x \in M$.

Lemma 3.2. Suppose that the family $\left\{\Psi(z): z \in \mathbb{C}_{+}\right\}$satisfies condition (3.1). Assume in addition that, for some $C \geq 1$ and some $a>0$,

$$
\left|\left\langle\Psi(t) f_{1}, f_{2}\right\rangle\right| \leq C e^{a t} e^{-\frac{r^{2}}{4 t}}\left\|f_{1}\right\|_{2}\left\|f_{2}\right\|_{2}, \quad \forall t>0,
$$

whenever $f_{i} \in L^{2}(M, d \mu)$, supp $f_{i} \subseteq B\left(x_{i}, r_{i}\right), i=1,2$, and $r=d\left(B\left(x_{1}, r_{1}\right), B\left(x_{2}, r_{2}\right)\right)$. Then the family $\left\{\Psi(z): z \in \mathbb{C}_{+}\right\}$satisfies condition (3.2).

Proof. Lemma 3.2 is a straightforward consequence of Proposition 2.2 and Lemma 3.1 .

Let us give an application of Lemma 3.2 by giving yet another example where DaviesGaffney estimates hold, namely Schrödinger semigroups with real potential. Suppose that $\Delta$ is the non-negative Laplace-Beltrami operator on a Riemannian manifold $M$ with Riemannian measure $\mu$ and geodesic distance $d$, and consider the operator $\Delta+\mathcal{V}$ acting on $C_{c}^{\infty}(M)$, where $\mathcal{V} \in L^{1}{ }_{\text {loc }}(M, d \mu)$. If we assume that $\Delta+\mathcal{V} \geq 0$ then we can define the Friedrichs extension of $\Delta+\mathcal{V}$, which with some abuse of notation we also denote by $\Delta+\mathcal{V}$ (see for example [23, Theorem 1.2.8].

Theorem 3.3. Suppose that $\Delta$ is the Laplace-Beltrami operator on a Riemannian manifold $M$, that $\mathcal{V} \in L^{1}{ }_{\text {loc }}(M, d \mu)$ and that $\Delta+\mathcal{V} \geq 0$ as a quadratic form. Then the semigroup $\left\{\Psi(z)=\exp (-z(\Delta+\mathcal{V})): z \in \mathbb{C}_{+}\right\}$satisfies condition (3.2).

Proof. We start our proof with the additional assumption $\mathcal{V} \geq 0$. For $f \in L^{2}(M, d \mu)$, $t>0, x \in M$, we put $f_{t}(x)=f(t, x)=\exp (-t(\Delta+\mathcal{V})) f(x)$. Let $\kappa>0$, and a function $\xi \in C^{\infty}(M)$, both to be chosen later, such that $|\nabla \xi| \leq \kappa$, where $\nabla$ is the Riemannian gradient on $M$. Next, as in [25, 35, 51, we consider the integral

$$
E(t)=\int_{M}|f(t, x)|^{2} e^{\xi(x)} d \mu(x) .
$$

Then

$$
\begin{aligned}
\frac{E^{\prime}(t)}{2} & =\operatorname{Re} \int_{M} \partial_{t} f(t, x) \overline{f(t, x)} e^{\xi(x)} d \mu(x)=-\operatorname{Re} \int_{M}\left((\Delta+\mathcal{V}) f_{t}\right) \overline{f_{t}} e^{\xi} d \mu \\
& =-\operatorname{Re} \int_{M}\left(\nabla f_{t} \cdot \nabla\left(f_{t} e^{\xi}\right)+\left|f_{t}\right|^{2} \mathcal{V} e^{\xi}\right) d \mu \\
& =-\operatorname{Re} \int_{M}\left(\left|\nabla f_{t}\right|^{2}+\nabla f_{t} \cdot f_{t} \nabla \xi+\left|f_{t}\right|^{2} \mathcal{V}\right) e^{\xi} d \mu \\
& \leq \int_{M}\left(-\left|\nabla f_{t}\right|^{2}+\left|\nabla f_{t}\right||\nabla \xi|\left|f_{t}\right|\right) e^{\xi} d \mu \\
& \leq \frac{1}{4} \int_{M}\left|f_{t}\right|^{2}|\nabla \xi|^{2} e^{\xi} d \mu \leq \frac{\kappa^{2} E(t)}{4}
\end{aligned}
$$

(note that the non-negativity of $\mathcal{V}$ is used in the first inequality). Hence $E(t) \leq \exp \left(\kappa^{2} t / 2\right) E(0)$. 
Consider now two disjoints open sets $U_{1}$ and $U_{2}$ in $M$. Choose $\xi=\kappa d\left(., U_{1}\right)$. One has $|\nabla \xi| \leq \kappa, \xi \equiv 0$ on $U_{1}$, and, for any $g \in L_{\text {loc }}^{2}(M, d \mu)$,

$$
\int_{U_{2}}|g|^{2} e^{\xi} d \mu \geq e^{\kappa r} \int_{U_{2}}|g|^{2} d \mu
$$

where $r=d\left(U_{1}, U_{2}\right)$. Hence if supp $f \subseteq U_{1}$ then, taking $g=f_{t}$,

$$
\int_{U_{2}}\left|f_{t}\right|^{2} d \mu \leq e^{-\kappa r} E(t) \leq \exp \left(\frac{\kappa^{2} t}{2}-\kappa r\right) E(0)=\exp \left(\frac{\kappa^{2} t}{2}-\kappa r\right) \int_{U_{1}}|f|^{2} d \mu .
$$

Choosing finally $\kappa=r / t$ we obtain

$$
\int_{U_{2}}|\Psi(t) f|^{2} d \mu \leq \exp \left(-\frac{r^{2}}{2 t}\right) \int_{U_{1}}|f|^{2} d \mu
$$

that is, for all $f \in L^{2}\left(U_{1}, d \mu\right)$,

$$
\sup _{g \in L^{2}\left(U_{2}, d \mu\right),\|g\|_{2}=1}|\langle\Psi(t) f, g\rangle|^{2}=\int_{U_{2}}|\Psi(t) f|^{2} d \mu \leq \exp \left(-\frac{r^{2}}{2 t}\right)\|f\|_{2}^{2},
$$

which yields (3.2). Next, we consider a potential $\mathcal{V} \in L_{\text {loc }}^{1}(M)$ such that $\Delta+\mathcal{V} \geq 0$. We put $\mathcal{V}_{a}(x)=\max \{\mathcal{V}(x),-a\}$ and $L_{a}=\Delta+\mathcal{V}_{a}$. When $a$ goes to $\infty$ then $L_{a}$ converges to $L=\Delta+\mathcal{V}$ in the strong resolvent sense (see [40, Theorem VIII.3.3, p.454] or [48, Theorem S.16 p.373]). Hence by [48, Theorem VIII.20, p.286] or by [40, Theorem VIII.3.11, p.459 and Theorem IX.2.16, p.504 ], $\exp \left(-t L_{a}\right) f$ converges to $\exp (-t L) f=\exp (-t(\Delta+\mathcal{V})) f$ for any $f \in L^{2}(M)$. Hence it is enough to prove (3.2) for a given $a \in \mathbb{R}$. Finally we note that $\mathcal{V}_{a}+a \geq 0$, thus it follows from the first part of the proof that

$$
\exp \left(-t\left(\Delta+\mathcal{V}_{a}+a\right)\right)=e^{-a t} \exp \left(-t\left(\Delta+\mathcal{V}_{a}\right)\right)
$$

satisfies condition (3.2). But this implies that the semigroup $\exp \left(-t\left(\Delta+\mathcal{V}_{a}\right)\right)$ satisfies condition (3.4) and Theorem 3.3 follows from Lemma 3.2

Remark: Note that the case $\mathcal{V}=0$ is allowed in Theorem 3.3, in other words it yields a proof of (3.2) for the Laplace-Beltrami operator on complete Riemannian manifolds.

\subsection{Finite speed propagation for the wave equation and Davies-Gaffney esti- mates.}

As a next application of the Phragmén-Lindelöf technique developed in Section 2, we show that, for self-adjoint operators, Davies-Gaffney estimates are equivalent to finite speed propagation property for the corresponding wave equation. This equivalence was proved, along the same lines, in [51]. The underlying idea is so basic to the development of the technique in the present paper that we shall repeat this proof. One can use this equivalence to obtain a very simple proof of the finite speed propagation property for a broad class of selfadjoint operators (see [51]). We start with recalling the notion of finite speed propagation property for the wave equation.

In this section, $(M, d, \mu)$ is again a metric measure space. We say that a non-negative self-adjoint operator $L$ satisfies the finite speed propagation property for solutions of the corresponding wave equation if

$$
\left\langle\cos (t \sqrt{L}) f_{1}, f_{2}\right\rangle=0
$$

for all $0<t<r$, open sets $U_{i} \subset M, f_{i} \in L^{2}\left(U_{i}, d \mu\right), i=1,2$, where $r=d\left(U_{1}, U_{2}\right)$. 
If $\cos (t \sqrt{L})$ is an integral operator with kernel $K_{t}$, then (3.5) simply means that supp $K_{t} \subseteq$ $\mathcal{D}_{t}$, that is $K_{t}\left(x_{1}, x_{2}\right)=0$ for all $\left(x_{1}, x_{2}\right) \notin \mathcal{D}_{t}$, where

$$
\mathcal{D}_{t}=\left\{\left(x_{1}, x_{2}\right) \in M \times M: d\left(x_{1}, x_{2}\right) \leq t\right\} .
$$

Theorem 3.4. Let $L$ be a self-adjoint non-negative operator acting on $L^{2}(M, d \mu)$. Then the finite speed propagation property (3.5) and Davies-Gaffney estimates (3.2) are equivalent.

Proof. Assume the Davies-Gaffney estimates. Fix two open sets $U_{1}, U_{2} \subset M$. Let $f_{i} \in$ $L^{2}\left(U_{i}, d \mu\right)$ for $i=1,2$. Define a function $F: \mathbb{C}_{+} \rightarrow \mathbb{C}$ by

$$
F(z)=\left\langle\exp (-z L) f_{1}, f_{2}\right\rangle .
$$

Since $\exp (-z L)$ is contractive on $L^{2}(M, d \mu), F$ is a bounded analytic function on $\mathbb{C}_{+}$and it satisfies (2.1) with

$$
B=\left\|f_{1}\right\|_{2}\left\|f_{2}\right\|_{2}
$$

In virtue of (3.2), $F$ satisfies (2.2) with

$$
a=0, \quad A=\left\|f_{1}\right\|_{2}\left\|f_{2}\right\|_{2}, \quad \gamma=\frac{r^{2}}{4} \quad \text { and } \quad r=d\left(U_{1}, U_{2}\right),
$$

thus, by Proposition 2.2 .

$$
|F(z)| \leq\left\|f_{1}\right\|_{2}\left\|f_{2}\right\|_{2} \exp \left(-r^{2} \operatorname{Re} \frac{1}{4 z}\right) .
$$

Then write, for $s>0$, the well-known Hadamard transmutation formula

$$
<\exp (-s L) f_{1}, f_{2}>=\int_{0}^{\infty}<\cos (t \sqrt{L}) f_{1}, f_{2}>\frac{e^{-\frac{t^{2}}{4 s}}}{\sqrt{\pi s}} d t .
$$

By the change of variable $t \rightarrow \sqrt{t}$ in integral (3.8) and changing $s$ to $1 /(4 s)$, we obtain

$$
s^{-1 / 2}<\exp \left(-\frac{L}{4 s}\right) f_{1}, f_{2}>=\int_{0}^{\infty}(\pi t)^{-1 / 2}<\cos (\sqrt{t} \sqrt{L}) f_{1}, f_{2}>e^{-s t} d t
$$

and by analytic continuation

$$
\zeta^{-1 / 2}<\exp \left(-\frac{L}{4 \zeta}\right) f_{1}, f_{2}>=\int_{0}^{\infty}(\pi t)^{-1 / 2}<\cos (\sqrt{t} \sqrt{L}) f_{1}, f_{2}>e^{-\zeta t} d t
$$

for $\zeta \in \mathbb{C}_{+}$. Consider the function $u(\zeta)=\zeta^{-1 / 2} F\left(\frac{1}{4 \zeta}\right)$. On the one hand, by (3.7),

$$
|u(\zeta)| \leq\left\|f_{1}\right\|_{2}\left\|f_{2}\right\|_{2}|\zeta|^{-1 / 2} \exp \left(-r^{2} \operatorname{Re} \zeta\right)
$$

On the other hand, by (3.9), $u$ is the Fourier-Laplace transform of the function

$$
v(t)=(\pi t)^{-1 / 2}<\cos (\sqrt{t} \sqrt{L}) f_{1}, f_{2}>.
$$

A suitable version of the Paley-Wiener theorem (see [39, Theorem 7.4.3, p.193]) shows that

$$
\text { supp } v \subseteq\left[r^{2}, \infty\right) \text {. }
$$

Thus $<\cos (\sqrt{t} \sqrt{L}) f_{1}, f_{2}>=0$ for all $0 \leq t \leq r^{2}$ and (3.5) is proved. 
Conversely if (3.5) holds, then by (3.8)

$$
\begin{aligned}
\left|<\exp (-s L) f_{1}, f_{2}>\right| & \leq \int_{0}^{\infty}\left|<\cos (t \sqrt{L}) f_{1}, f_{2}>\right| \frac{e^{-\frac{t^{2}}{4 s}}}{\sqrt{\pi s}} d t \\
& =\int_{r}^{\infty}\left|<\cos (t \sqrt{L}) f_{1}, f_{2}>\right| \frac{e^{-\frac{t^{2}}{4 s}}}{\sqrt{\pi s}} d t \\
& \leq\left\|f_{1}\right\|_{2}\left\|f_{2}\right\|_{2} \int_{r}^{\infty} \frac{e^{-\frac{t^{2}}{4 s}}}{\sqrt{\pi s}} d t \\
& \leq e^{-\frac{r^{2}}{4 s}}\left\|f_{1}\right\|_{2}\left\|f_{2}\right\|_{2} .
\end{aligned}
$$

\section{From on-Diagonal bounds to Gaussian bounds}

In this section, $(M, d, \mu)$ is again a metric measure space. Let $L$ be a non-negative self-adjoint operator acting on $L^{2}(M, d \mu)$; recall that the semigroup of operators $\Psi(z)=$ $\exp (-z L), z \in \mathbb{C}_{+}$, is contractive on $L^{2}(M, d \mu)$, in other words it satisfies condition (3.1).

Our basic observation is the following : if $p_{z}$ is the kernel associated with $\exp (-z L)$, the estimate

$$
p_{t}(x, x) \leq K t^{-D / 2}, \forall t>0
$$

can be reformulated as

$$
\|\exp (-t L)\|_{1 \rightarrow \infty} \leq K t^{-D / 2}, \forall t>0
$$

which yields

$$
\|\exp (-z L)\|_{1 \rightarrow \infty} \leq K(\operatorname{Re} z)^{-D / 2}, \forall z \in \mathbb{C}_{+}
$$

that is, in terms of a bilinear estimate,

$$
\left|<\exp (-z L) f_{1}, f_{2}>\right| \leq K(\operatorname{Re} z)^{-D / 2}\left\|f_{1}\right\|_{1}\left\|f_{2}\right\|_{1}, \forall z \in \mathbb{C}_{+}, f_{1}, f_{2} \in L^{1}(M, d \mu) .
$$

On the other hand, Davies-Gaffney says that

$$
\left|\left\langle\exp (-t L) f_{1}, f_{2}\right\rangle\right| \leq \exp \left(-\frac{r^{2}}{4 t}\right)\left\|f_{1}\right\|_{2}\left\|f_{2}\right\|_{2}
$$

for all $t>0, f_{1}, f_{2} \in L^{2}(M, d \mu)$, supported respectively in $U_{1}, U_{2}$, with $r=d\left(U_{1}, U_{2}\right)$. With these ingredients and the global $L^{2}$ bound on the complex half-plane

$$
\left|<\exp (-z L) f_{1}, f_{2}>\right| \leq\left\|f_{1}\right\|_{2}\left\|f_{2}\right\|_{2}, \forall z \in \mathbb{C}_{+}, f_{1}, f_{2} \in L^{2}(M, d \mu),
$$

the suitable Phragmén-Lindelöf type lemma yields

$$
\left|\left\langle\exp (-z L) f_{1}, f_{2}\right\rangle\right| \leq e K(\operatorname{Re} z)^{-D / 2}\left(\operatorname{Re} \frac{r^{2}}{4 z}\right)^{D / 2} \exp \left(-\operatorname{Re} \frac{r^{2}}{4 z}\right)\left\|f_{1}\right\|_{1}\left\|f_{2}\right\|_{1},
$$

for $z$ in the relevant region of the half-plane and all $f_{1}, f_{2} \in L^{1}(M, d \mu)$, supported in $U_{1}, U_{2}$, with $r=d\left(U_{1}, U_{2}\right)$. Taking for $U_{1}, U_{2}$ balls that shrink around $x$ and $y$, one obtains, for $\operatorname{Re} \frac{d^{2}(x, y)}{4 z}$ large enough, the desired estimate

$$
\left|p_{z}(x, y)\right| \leq e K(\operatorname{Re} z)^{-D / 2}\left(\operatorname{Re} \frac{d^{2}(x, y)}{4 z}\right)^{D / 2} \exp \left(-\operatorname{Re} \frac{d^{2}(x, y)}{4 z}\right) .
$$


Let us see this in more detail, and then in more general situations.

\subsection{Polynomial decay.}

Assume

It follows that

$$
\|\exp (-t L)\|_{1 \rightarrow \infty} \leq K t^{-D / 2}, \forall t>0
$$

$$
\|\exp (-z L)\|_{1 \rightarrow \infty} \leq K(\operatorname{Re} z)^{-D / 2}, \forall z \in \mathbb{C}_{+} .
$$

Indeed, for $t>0, s \in \mathbb{R}$,

$$
\begin{aligned}
\|\exp (-(t+i s) L)\|_{1 \rightarrow \infty} & \leq\|\exp (-t L / 2)\|_{1 \rightarrow 2}\|\exp (-i s L)\|_{2 \rightarrow 2}\|\exp (-t L / 2)\|_{2 \rightarrow \infty} \\
& =\|\exp (-t L / 2)\|_{1 \rightarrow 2}^{2}\|\exp (-i s L)\|_{2 \rightarrow 2} \\
& =\|\exp (-t L)\|_{1 \rightarrow \infty}\|\exp (-i s L)\|_{2 \rightarrow 2} \\
& \leq K t^{-D / 2}
\end{aligned}
$$

using the well-known equality $\left\|T^{*} T\right\|_{1 \rightarrow \infty}=\|T\|_{1 \rightarrow 2}^{2}$.

In particular, by [29, Theorem 6, p.503], $\exp (-z L)$ is an integral operator for all $z \in \mathbb{C}_{+}$. This means there exists a measurable kernel, which we denote by $p_{z}(x, y)$, such that

$$
[\exp (-z L) f](x)=\int_{M} p_{z}(x, y) f(y) d \mu(y), \text { for a.e. } x \in M .
$$

Before we start discussing Gaussian bounds, let us state another straightforward consequence of [29, Theorem 6, p.503], which we are going to use frequently in the sequel. A linear operator $S$ is bounded from $L^{1}(M, d \mu)$ to $L^{\infty}(M, d \mu)$ if and only if it is an integral operator with kernel $p(x, y)$ such that $\operatorname{esssup}_{x \in M, y \in M}|p(x, y)|$ is finite, in which case

$$
\operatorname{esssup}_{x \in M, y \in M}|p(x, y)|=\|S\|_{1 \rightarrow \infty} .
$$

More precisely, we have the following :

Claim 1. Let $U_{1}, U_{2}$ be open subsets of $M$. If $p(x, y)$ is the kernel of a linear operator $S: L^{1}(M, d \mu) \rightarrow L^{\infty}(M, d \mu)$, then

$$
\operatorname{esssup}_{x \in U_{1}, y \in U_{2}}|p(x, y)|=\sup \left\{\left|\left\langle S f_{1}, f_{2}\right\rangle\right|:\left\|f_{1}\right\|_{L^{1}\left(U_{1}, d \mu\right)}=\left\|f_{2}\right\|_{L^{1}\left(U_{2}, d \mu\right)}=1\right\} .
$$

To complete the last step of the argument we sketched above, namely to pass from estimates on arbitrarily small balls to pointwise estimates, we need to assume space continuity of the kernel under consideration, which is the case in most concrete situations, but not in general. We shall assume from now on that for every $z \in \mathbb{C}_{+}$the kernel $p_{z}$ is a continuous complex-valued function defined on $M \times M{ }^{3}$ As a consequence, we can replace the essential suprema by suprema in the above expressions, and also record the following :

If $p_{t}(x, y)$ is the kernel of $\exp (-t L)$, a well-known argument using the semigroup property and the fact that $p_{t}(y, x)=\overline{p_{t}(x, y)}$ (see the proof of (4.15) below) shows further that

$$
\|\exp (-t L)\|_{1 \rightarrow \infty}=\sup _{x, y \in M}\left|p_{t}(x, y)\right|=\sup _{x \in M} p_{t}(x, x) .
$$

We can now state the general version of Theorem 1.1. An even more general version will be given in Corollary 4.4 below, at the expense of a slightly more complicated proof.

\footnotetext{
${ }^{3}$ For an interesting discussion about continuity properties of a general heat kernel, see [36. On the other hand, Brian Davies told us about a folklore example of a decent Schrödinger operator on $\mathbb{R}$ whose on-diagonal values of the kernel are null on a countable dense subset.
} 
Theorem 4.1. Assume that $(M, d, \mu, L)$ satisfies the Davies-Gaffney condition (3.2). If, for some $K$ and $D>0$,

$$
p_{t}(x, x) \leq K t^{-D / 2}, \forall t>0, x \in M
$$

then

$$
\left|p_{z}(x, y)\right| \leq e K(\operatorname{Re} z)^{-D / 2}\left(1+\operatorname{Re} \frac{d^{2}(x, y)}{4 z}\right)^{D / 2} \exp \left(-\operatorname{Re} \frac{d^{2}(x, y)}{4 z}\right)
$$

for all $z \in \mathbb{C}_{+}, x, y \in M$.

Proof. Fix $x, y \in M$, and for $d(x, y)>2 s>0$ define a bounded analytic function $F: \mathbb{C}_{+} \rightarrow$ $\mathbb{C}$ as in (3.6) by the formula

$$
F(z)=\left\langle\exp (-z L) f_{1}, f_{2}\right\rangle,
$$

where $f_{1} \in L^{1}(B(x, s), d \mu) \cap L^{2}(B(x, s), d \mu), f_{2} \in L^{1}(B(y, s), d \mu) \cap L^{2}(B(y, s), d \mu)$ and $\left\|f_{1}\right\|_{1}=\left\|f_{2}\right\|_{1}=1$. In virtue of Davies-Gaffney estimates (3.2) and (3.1), $F$ satisfies (2.8) and (2.9) with

$$
\gamma=r^{2} / 4, \text { where } r=d(x, y)-2 s, \quad \text { and } \quad A=\left\|f_{1}\right\|_{2}\left\|f_{2}\right\|_{2}<\infty .
$$

Assumption (4.4) yields, through (4.3) and (4.1),

$$
|F(z)|=\left|\left\langle\exp (-z L) f_{1}, f_{2}\right\rangle\right| \leq K(\operatorname{Re} z)^{-D / 2}, \forall z \in \mathbb{C}_{+},
$$

so that $F$ satisfies (2.10) with $\nu=D$ and $B=K r^{-D}$. By Proposition 2.3

$$
\begin{aligned}
|F(z)|=\left|\left\langle\exp (-z L) f_{1}, f_{2}\right\rangle\right| & \leq e K r^{-D}\left(\frac{r^{2}}{2|z|}\right)^{D} \exp \left(-\operatorname{Re} \frac{r^{2}}{4 z}\right) \\
& =e K\left(\frac{r^{2}}{4|z|^{2}}\right)^{D / 2} \exp \left(-\operatorname{Re} \frac{r^{2}}{4 z}\right) \\
& =e K(\operatorname{Re} z)^{-D / 2}\left(\operatorname{Re} \frac{r^{2}}{4 z}\right)^{D / 2} \exp \left(-\operatorname{Re} \frac{r^{2}}{4 z}\right)
\end{aligned}
$$

for all $z \in \mathcal{C}_{r^{2} / 4}$.

Hence by Claim 1

$$
\begin{aligned}
\left|p_{z}(x, y)\right| & \leq \sup _{x^{\prime} \in B(x, s)} \sup _{y^{\prime} \in B(y, s)}\left|p_{z}\left(x^{\prime}, y^{\prime}\right)\right| \\
& =\sup \left\{\left\langle\exp (-z L) f_{1}, f_{2}\right\rangle \mid:\left\|f_{1}\right\|_{L^{1}(B(x, s), d \mu)}=\left\|f_{2}\right\|_{L^{1}(B(y, s), d \mu)}=1\right\} \\
& \leq e K(\operatorname{Re} z)^{-D / 2}\left(\operatorname{Re} \frac{r^{2}}{4 z}\right)^{D / 2} \exp \left(-\operatorname{Re} \frac{r^{2}}{4 z}\right)
\end{aligned}
$$

for all $z \in \mathcal{C}_{r^{2} / 4}$.

Letting $s$ go to 0 we obtain (4.5) for $\operatorname{Re} \frac{d^{2}(x, y)}{4 z} \geq 1$. Finally for $\operatorname{Re} \frac{d^{2}(x, y)}{4 z}<1$, (4.5) is a straightforward consequence of (4.4). Indeed in that case the Gaussian correction term satisfies $\exp \left(-\operatorname{Re} \frac{d^{2}(x, y)}{4 z}\right)>e^{-1}$, and the estimate (4.5) follows from

$$
\left|p_{z}(x, y)\right| \leq K(\operatorname{Re} z)^{-D / 2}
$$

which in turn follows from (4.1). 
Now for the general version of Theorem 1.2. Again, an even more general version will be given in Theorem 4.6 below.

Theorem 4.2. Assume that $(M, d, \mu, L)$ satisfies the Davies-Gaffney condition (3.2). Next suppose that

$$
\left|p_{z}(x, y)\right| \leq K|z|^{-D / 2}, \forall z \in \mathbb{C}_{+}, x, y \in M,
$$

for some $K$ and $D>0$. Then

$$
\left|p_{z}(x, y)\right| \leq e K|z|^{-D / 2} \exp \left(-\operatorname{Re} \frac{d^{2}(x, y)}{4 z}\right)
$$

for all $z \in \mathbb{C}_{+}, x, y \in M$.

Proof. One starts as in the proof of Theorem 4.1 Then condition (4.6) yields

$$
F(z):=\left|\left\langle\exp (-z L) f_{1}, f_{2}\right\rangle\right| \leq K|z|^{-D / 2} .
$$

Choosing $g$ so that $|\exp (g(z))|=|z|^{D / 2}$ and taking $B=K, \gamma=r^{2} / 4, \beta=0, \nu=0$ in Proposition 2.4, one obtains

$$
\left|\left\langle\exp (-z L) f_{1}, f_{2}\right\rangle\right| \leq K|z|^{-D / 2} \exp \left(-\operatorname{Re} \frac{r^{2}}{4 z}\right)
$$

and the rest of the proof is as before.

Remarks: The fact that an on-diagonal estimate for the heat kernel implies an off-diagonal estimate is of course not new. See for example [23, Theorem 3.2.7, p.89] for the real time estimate and [23, Theorem 3.4.8, p.103] for the complex time estimate. Note however, that the results obtained in [23] are less precise than (4.5) because they involve $4+\varepsilon$ instead of 4 in the exponential factor. To our knowledge, the estimates (4.5) with 4 as an exponential factor are new for complex time. On the other hand, for real time and diffusion semigroups, estimates (4.5) were obtained in [26] (see also [16]).

For $z=t \in \mathbb{R}_{+}$, the estimates (4.5) can still be improved. It is possible to prove that

$$
\left|p_{t}(x, y)\right| \leq C t^{-D / 2}\left(1+\frac{d^{2}(x, y)}{4 t}\right)^{(D-1) / 2} \exp \left(-\frac{d^{2}(x, y)}{4 t}\right)
$$

(see [49]), and this is sharp due to [45]. It is an interesting question why our results here (and results in [26] and [16]) give weaker estimates with $D / 2$ instead of $(D-1) / 2$. It is so because in our proof we do not use the fact that the family of operators under consideration is a semigroup generated by a self-adjoint operator; for more on this, see the discussion in the remark at the end of Section 4.5 .

Suppose now that the self-adjoint contractive semigroup $\exp (-t L)$ on $L^{2}(M, d \mu)$ is in addition uniformly bounded on $L^{\infty}(M, d \mu)$, which includes the case of the heat semigroup on a complete Riemannian manifold, since it is submarkovian. Suppose also that estimates (4.4) hold and that

$$
\|\exp (i s L)\|_{1 \rightarrow \infty} \leq C|s|^{-D / 2}, \quad \forall s \in \mathbb{R} \backslash\{0\} .
$$


Then the semigroup $\exp (-z L)$ satisfies condition (4.6), hence the corresponding heat kernel satisfies estimates (4.7). Indeed, by (4.8) for all $t>0, s \in \mathbb{R}$,

$$
\|\exp (-(t+i s) L)\|_{1 \rightarrow \infty} \leq\|\exp (-t L / 2)\|_{\infty \rightarrow \infty}\|\exp (-i s L)\|_{1 \rightarrow \infty} \leq C|s|^{-D / 2} .
$$

Together with (4.1) this yields

$$
\|\exp (-(t+i s) L)\|_{1 \rightarrow \infty} \leq C \min \left\{t^{-D / 2},|s|^{-D / 2}\right\} \leq C^{\prime}|t+i s|^{-D / 2}
$$

for all $t>0, s \in \mathbb{R}$ (as a matter of fact, (4.6) is equivalent to the conjunction of (4.4) and (4.8) $)$. This shows that Gaussian bounds without an additional polynomial correction factor are a necessary condition for (4.8) to hold. Let us observe that estimates (4.8) play an essential role in studying Strichartz type estimates (see for example [4]).

4.2. The doubling case. Let $(M, d, \mu)$ be a metric measure space as above, and let $p_{z}$, $z \in \mathbb{C}_{+}$be a continuous heat kernel corresponding to a non-negative self-adjoint operator $L$ on $L^{2}(M, d \mu)$.

One says that $(M, d, \mu)$ satisfies the doubling property if there exists $C>0$ such that

$$
\mu(B(x, 2 r)) \leq C \mu(B(x, r)), \quad \forall r>0, x \in M .
$$

If this is the case, there exist $C, \delta>0$ such that

$$
\frac{\mu(B(x, s))}{\mu(B(x, r))} \leq C\left(\frac{s}{r}\right)^{\delta}, \quad \forall s \geq r>0, x \in M .
$$

In such a situation, the most natural on-diagonal estimates for heat kernels are of the type

$$
p_{t}(x, x) \leq \frac{C}{\mu(B(x, \sqrt{t}))}, \forall t>0, x \in M
$$

(see for instance [35]).

We are going to consider estimates of a similar form, but where the quantity $\mu(B(x, \sqrt{t}))$ will be replaced by a function $V$ of $x$ and $t$ that is not necessarily connected with the volume of balls.

We shall assume that $V: M \times \mathbb{R}_{+} \rightarrow \mathbb{R}_{+}$is non-decreasing in the second variable, that is $V(x, s) \leq V(x, r)$ for all $x \in M$ and all $0<s \leq r$, and that it satisfies the doubling condition

$$
\frac{V(x, s)}{V(x, r)} \leq K^{\prime}\left(\frac{s}{r}\right)^{\delta}
$$

for all $s \geq r>0$ and all $x \in M$, and some constants $\delta \geq 0$ and $K^{\prime} \geq 1$. Finally we shall assume that $V(x, t)$ is a continuous function of $x$.

We shall then consider the on-diagonal estimate

$$
p_{t}(x, x) V(x, \sqrt{t}) \leq 1, \forall x \in M, t>0 .
$$

One should compare the following result with [51, Theorem 4], which yields a slightly more precise estimate for real time. 
Theorem 4.3. Assume that $(M, d, \mu, L)$ satisfies the Davies-Gaffney condition (3.2). Next assume that the corresponding heat kernel $p_{z}$ is continuous and satisfies the on-diagonal estimate (4.12) with $V$ satisfying the doubling condition (4.11). Then

$$
\left|p_{z}(x, y)\right| \leq \frac{e K^{\prime}}{\sqrt{V\left(x, \frac{d(x, y)}{2}\right) V\left(y, \frac{d(x, y)}{2}\right)}}\left(\frac{d^{2}(x, y)}{4|z|}\right)^{\delta} \exp \left(-\operatorname{Re} \frac{d^{2}(x, y)}{4 z}\right)
$$

for all $z \in \mathbb{C}_{+}, x, y \in M$ such that $\operatorname{Re} \frac{d^{2}(x, y)}{4 z} \geq 1$. For all $z \in \mathbb{C}_{+}, x, y \in M$, and in particular if $\operatorname{Re} \frac{d^{2}(x, y)}{4 z}<1$, one has

$$
\left|p_{z}(x, y)\right| \leq \frac{1}{\sqrt{V(x, \sqrt{\operatorname{Re} z}) V(y, \sqrt{\operatorname{Re} z})}} .
$$

Proof. For all $z \in \mathbb{C}_{+}, x, y \in M$, one has

$$
\left|p_{z}(x, y)\right| \leq \sqrt{p_{\operatorname{Re} z}(x, x) p_{\operatorname{Re} z}(y, y)} .
$$

Indeed,

$$
\begin{aligned}
\left|p_{z}(x, y)\right| & =\left|\int_{M} p_{z / 2}(x, u) p_{z / 2}(u, y) d \mu(u)\right| \\
& \leq\left(\int_{M}\left|p_{z / 2}(x, u)\right|^{2} d \mu(u)\right)^{1 / 2}\left(\int_{M}\left|p_{z / 2}(u, y)\right|^{2} d \mu(u)\right)^{1 / 2} \\
& =\left(\int_{M} p_{z / 2}(x, u) p_{\bar{z} / 2}(u, x) d \mu(u)\right)^{1 / 2}\left(\int_{M} p_{\bar{z} / 2}(y, u) p_{z / 2}(u, y) d \mu(u)\right)^{1 / 2} \\
& \leq \sqrt{p_{\operatorname{Re} z}(x, x) p_{\operatorname{Re} z}(y, y)}
\end{aligned}
$$

In the second equality above, we have used the fact that, since $L$ is self-adjoint, $\overline{p_{z}(x, y)}=$ $p_{\bar{z}}(y, x)$. Together with (4.12), this yields (4.14).

For a function $W: M \rightarrow \mathbb{C}$, we denote by $\mathbf{m}_{W}$ the operator of multiplication by $W$, that is

$$
\left(\mathbf{m}_{W} f\right)(x)=W(x) f(x),
$$

and if $W: M \times \mathbb{R}_{+} \rightarrow \mathbb{C}$ then for $r \in \mathbb{R}_{+}$we set

$$
\left(\mathbf{m}_{W(\cdot, r)} f\right)(x)=W(x, r) f(x) .
$$

Let us now set $W(x, r)=\sqrt{V(x, r)}$. Similarly as in the proof of Theorem 4.1, fix $x, y \in M$ and for $d(x, y)>2 s>0$ set $r=d(x, y)-2 s$. Then define a bounded analytic function $F: \mathbb{C}_{+} \rightarrow \mathbb{C}$ by the formula

$$
F(z)=\left\langle\exp (-z L) \mathbf{m}_{W\left(\cdot, \frac{r}{2}\right)} f_{1}, \mathbf{m}_{W\left(\cdot, \frac{r}{2}\right)} f_{2}\right\rangle,
$$

where

$f_{1} \in L^{1}(B(x, s), d \mu) \cap L^{2}(B(x, s), V(\cdot, r / 2) d \mu), f_{2} \in L^{1}(B(y, s), d \mu) \cap L^{2}(B(y, s), V(\cdot, r / 2) d \mu)$, and $\left\|f_{1}\right\|_{1}=\left\|f_{2}\right\|_{1}=1$. In virtue of (3.2) and (3.1), $F$ satisfies (2.8) and (2.9) with

$$
A=\left\|\mathbf{m}_{W\left(\cdot, \frac{r}{2}\right)} f_{1}\right\|_{2}\left\|\mathbf{m}_{W\left(\cdot, \frac{r}{2}\right)} f_{2}\right\|_{2}<\infty \text { and } \gamma=r^{2} / 4 \text {. }
$$


Note that, for $z \in \mathcal{C}_{\gamma}$,

$$
\gamma \geq\left(\operatorname{Re} \frac{1}{z}\right)^{-1} \geq \operatorname{Re} z
$$

hence $r / 2 \geq \sqrt{\operatorname{Re} z}$. Now by (4.12), (4.17) and (4.11),

$$
\begin{aligned}
|F(z)| & =\left|\int_{M} \int_{M} p_{z}\left(x^{\prime}, y^{\prime}\right) W\left(y^{\prime}, \frac{r}{2}\right) f_{1}\left(y^{\prime}\right) W\left(x^{\prime}, \frac{r}{2}\right) f_{2}\left(x^{\prime}\right) d \mu\left(y^{\prime}\right) d \mu\left(x^{\prime}\right)\right| \\
& \leq \sup _{x^{\prime}, y^{\prime} \in M} W\left(x^{\prime}, \frac{r}{2}\right)\left|p_{z}\left(x^{\prime}, y^{\prime}\right)\right| W\left(y^{\prime}, \frac{r}{2}\right) \\
& \leq \sup _{x^{\prime}, y^{\prime} \in M} \sqrt{\frac{V\left(x^{\prime}, \frac{r}{2}\right) V\left(y^{\prime}, \frac{r}{2}\right)}{V\left(x^{\prime}, \sqrt{\operatorname{Re} z}\right) V\left(y^{\prime}, \sqrt{\operatorname{Re} z}\right)}} \leq K^{\prime}\left(\frac{r}{2 \sqrt{\operatorname{Re} z}}\right)^{\delta}=K^{\prime} 2^{-\delta}\left(\frac{\operatorname{Re} z}{r^{2}}\right)^{-\delta / 2}
\end{aligned}
$$

so that $F$ satisfies (2.10) with $B=K^{\prime} 2^{-\delta}$ and $\nu=\delta$. By Proposition 2.3,

$$
|F(z)| \leq e K^{\prime} 2^{-\delta}\left(\frac{r^{2}}{2|z|}\right)^{\delta} \exp \left(-\operatorname{Re} \frac{r^{2}}{4 z}\right)
$$

for all $z \in \mathcal{C}_{r^{2} / 4}$. Note that $L^{1}(B(x, s), d \mu) \cap L^{2}(B(x, s), V(\cdot, r / 2) d \mu)$ is dense in $L^{1}(B(x, s), d \mu)$, so by Claim 11.

$$
\begin{aligned}
V^{1 / 2}\left(x, \frac{r}{2}\right)\left|p_{z}(x, y)\right| V^{1 / 2}\left(y, \frac{r}{2}\right) & \leq \sup _{x^{\prime} \in B(x, s)} \sup _{y^{\prime} \in B(y, s)} V^{1 / 2}\left(x^{\prime}, \frac{r}{2}\right)\left|p_{z}\left(x^{\prime}, y^{\prime}\right)\right| V^{1 / 2}\left(y^{\prime}, \frac{r}{2}\right) \\
& =\sup \left\{|F(z)|:\left\|f_{1}\right\|_{L^{1}(B(x, s), d \mu)}=\left\|f_{2}\right\|_{L^{1}(B(y, s), d \mu)}=1\right\} \\
& \leq e K^{\prime}\left(\frac{r^{2}}{4|z|}\right)^{\delta} \exp \left(-\operatorname{Re} \frac{r^{2}}{4 z}\right)
\end{aligned}
$$

for all $z \in \mathcal{C}_{r^{2} / 4}$. Letting $s \rightarrow 0$, we obtain the estimate (4.13) for $z \in \mathcal{C}_{d^{2}(x, y) / 4}$.

Note that taking

$$
V(x, r)=K^{-1} r^{D}, r>0, x \in M,
$$

one sees that Theorem 4.1 is a particular case of Theorem 4.3.

The estimate in the following corollary is less precise than the one in Theorem 4.3, but its algebraic form is convenient for calculations and it is enough for most applications; also, it can be compared with the case $m=2$ of the estimates in [14, Proposition 4.1]. The improvement with respect to [14 is that the constant inside the exponential is optimal, at the expense of a necessary polynomial correction factor. To state the result, we put $\theta=\arg z$ for all $z \in \mathbb{C}_{+}$, so that $\cos \theta=\frac{\operatorname{Re} z}{|z|}$.

Corollary 4.4. Under the assumptions of Theorem 4.3.

$$
\left|p_{z}(x, y)\right| \leq \frac{e K^{\prime}\left(1+\operatorname{Re} \frac{d^{2}(x, y)}{4 z}\right)^{\delta}}{\sqrt{V\left(x, \sqrt{\frac{|z|}{\cos \theta}}\right) V\left(y, \sqrt{\frac{|z|}{\cos \theta}}\right)}} \exp \left(-\operatorname{Re} \frac{d^{2}(x, y)}{4 z}\right) \frac{1}{(\cos \theta)^{\delta}}
$$

for all $z \in \mathbb{C}_{+}, x, y \in M$. 
Proof. Note that $\operatorname{Re} z^{-1}=|z|^{-1} \cos \theta$, hence

$$
\frac{d^{2}(x, y)}{4|z|} \cos \theta=\operatorname{Re} \frac{d^{2}(x, y)}{4 z}, z \in \mathbb{C}_{+} .
$$

Moreover, if $z \in \mathcal{C}_{d^{2}(x, y) / 4}$,

$$
\frac{d(x, y)}{2} \geq\left(\operatorname{Re} z^{-1}\right)^{-1 / 2}=(|z| / \cos \theta)^{1 / 2}
$$

hence

$$
V\left(x, \frac{d(x, y)}{2}\right) \geq V\left(x, \sqrt{\frac{|z|}{\cos \theta}}\right)
$$

Therefore

$$
\begin{aligned}
& \frac{1}{\sqrt{V\left(x, \frac{d(x, y)}{2}\right) V\left(y, \frac{d(x, y)}{2}\right)}}\left(\frac{d^{2}(x, y)}{4|z|}\right)^{\delta} \exp \left(-\operatorname{Re} \frac{d^{2}(x, y)}{4 z}\right) \\
\leq & \frac{1}{\sqrt{V\left(x, \sqrt{\frac{|z|}{\cos \theta}}\right) V\left(y, \sqrt{\frac{|z|}{\cos \theta}}\right.}}\left(\operatorname{Re} \frac{d^{2}(x, y)}{4 z}\right)^{\delta} \exp \left(-\operatorname{Re} \frac{d^{2}(x, y)}{4 z}\right) \frac{1}{(\cos \theta)^{\delta}}
\end{aligned}
$$

and for $z \in \mathcal{C}_{d^{2}(x, y) / 4}$, (4.18) follows from (4.13). Finally we note that by (4.11)

$$
V\left(x, \sqrt{\frac{|z|}{\cos \theta}}\right) \leq K^{\prime} V(x, \sqrt{|z| \cos \theta})(\cos \theta)^{-\delta}=K^{\prime} V(x, \sqrt{\operatorname{Re} z})(\cos \theta)^{-\delta}
$$

so that, for $z \notin \mathcal{C}_{d^{2}(x, y) / 4}$, since $\exp \left(-\operatorname{Re} \frac{d^{2}(x, y)}{4 z}\right) \geq e^{-1}$, 4.18) is a straightforward consequence of (4.14).

It is certainly an interesting feature of Corollary 4.4 that it yields estimates valid for time ranging in the whole right half-plane, and that it does not require $V$ to be tied to the volume. Let us however observe the following particular case of our result, for real time and estimates involving the volume growth function. It also follows from [33, Proposition 5.2], but our proof is more direct, as it does not go through a Faber-Krahn type inequality.

Corollary 4.5. Let $p_{t}, t>0$, be the heat kernel on a complete Riemannian manifold $M$, with Riemannian measure $\mu$ and geodesic distance $d$. Let $V(x, r)$ denote $\mu(B(x, r))$, for $r>0, x \in M$. Assume that $M$ satisfies the doubling property, more precisely let $K^{\prime}, \delta>0$ be such that (4.11) is satisfied. Suppose that

$$
p_{t}(x, x) \leq \frac{K}{V(x, \sqrt{t})}, \forall t>0, x \in M,
$$

for some $K>0$. Then

$$
p_{t}(x, y) \leq \frac{e K^{\prime} K\left(1+\frac{d^{2}(x, y)}{4 t}\right)^{\delta}}{\sqrt{V(x, \sqrt{t}) V(y, \sqrt{t})}} \exp \left(-\frac{d^{2}(x, y)}{4 t}\right)
$$

for all $t>0, x, y \in M$. 
Let us now consider the case where the heat kernel satisfies upper and lower estimates of the type

$$
p_{t}(x, y) \simeq \frac{1}{\mu\left(B\left(x, t^{1 / \beta}\right)\right)} \exp \left(-\left(\frac{d^{\beta}(x, y)}{t}\right)^{\frac{1}{\beta-1}}\right), \forall t>0, x, y \in M .
$$

This may happen when $(M, d, \mu, L)$ is a fractal space, endowed with a natural metric, measure and Laplacian, for all values of $\beta$ between 2 and $\delta+1$, where $\delta$ is the exponent in the doubling condition (4.11); see for instance 6]. In such situations, usually, $\beta>2$. Let us now choose $V(x, t)=\mu\left(B\left(x, t^{2 / \beta}\right)\right.$, which is obviously a doubling function. From (4.20), $p_{t}$ satisfies (4.12), but it cannot satisfy

$$
p_{t}(x, y) \leq \frac{C}{V(x, \sqrt{t})} \exp \left(-c \frac{d^{2}(x, y)}{t}\right)=\frac{C}{\mu\left(B\left(x, t^{1 / \beta}\right)\right)} \exp \left(-\frac{d^{2}(x, y)}{4 t}\right)
$$

since this is not compatible with the lower bound in (4.20). In view of Corollary 4.4, the only possible conclusion is that such a space $(M, d, \mu, L)$ does not satisfy Davies-Gaffney estimates, nor, according to (3.4), the finite speed propagation property for the wave equation. We owe this remark to Alexander Teplyaev. There is no contradiction with the fact that local Dirichlet forms do give rise to Davies-Gaffney estimates with respect to an intrinsic distance : in the case of fractals, this distance degenerates, see the discussion in [38, Section $3.2]$.

Finally let us discuss one more version of pointwise Gaussian estimates. Here we do not need to consider any kind of doubling property. The following result has some similarity with Corollary 5.5 of 35], in the sense that, in assumption (4.21) below, $x, y$ do not range in the whole space $M$, but only in two fixed regions $U_{1}, U_{2}$. However, restricting our assumption to two fixed points $x, y$ as in [35] seems to raise technical difficulties that we are not going to face here.

Theorem 4.6. Assume that $(M, d, \mu, L)$ satisfies the Davies-Gaffney condition (3.2). Let $U_{1}$ and $U_{2}$ be open subsets of $M$. Suppose that

$$
\left|p_{z}(x, y)\right| \leq \exp (-\operatorname{Re} g(z)), \quad \forall z \in \mathbb{C}_{+}, x \in U_{1}, y \in U_{2},
$$

where $g$ is analytic on $\mathbb{C}_{+}$and satisfies the growth condition (2.14) with $\gamma=\frac{r^{2}}{4}$ and $r=$ $d\left(U_{1}, U_{2}\right)$. Then

$$
\left|p_{z}(x, y)\right| \leq \exp \left(1-\operatorname{Re} g(z)-\operatorname{Re} \frac{r^{2}}{4 z}\right), \quad \forall z \in \mathbb{C}_{+}, x \in U_{1}, y \in U_{2} .
$$

Remarks:

- It may look surprising that the growth constraint on $g$ depends on $U_{1}, U_{2}$. This may be understood as follows : suppose a factor $\exp \left(-\operatorname{Re} \frac{r^{2}}{4 z}\right)$ is already present in estimate (4.21) (which corresponds to $\beta=1$, a situation hopefully forbidden by (2.14)) ; then one can certainly not multiply again the estimate by this factor!

- Theorem 4.6 is a generalization of Theorem 4.2, as one can see by taking $g(z)=\frac{D}{2} \log z-$ $\log K$. 

form

-In principle, one could use Theorem 4.6 to add a Gaussian factor to estimates of the

$$
\left|p_{z}(x, y)\right| \leq \frac{1}{|V(x, y, z)|}, \quad \forall z \in \mathbb{C}_{+}, x, y \in M,
$$

where $V$ is analytic in $z$ with a certain uniformity in $x, y$. We will not pursue this direction because of the lack of relevant examples.

-Note that our result allows to some extent rapid growth at zero. In particular, it might be interesting to investigate the connection with [7, Theorem 3.1].

Proof. Once again we follow the idea of the proof of Theorem 4.1 and define a bounded analytic function $F: \mathbb{C}_{+} \rightarrow \mathbb{C}$ by the formula

$$
F(z)=\left\langle\exp (-z L) f_{1}, f_{2}\right\rangle,
$$

where $f_{i} \in L^{2}\left(U_{i}, d \mu\right) \cap L^{1}\left(U_{i}, d \mu\right)$ and $\left\|f_{1}\right\|_{1}=\|f\|_{2}=1$. In virtue of (3.2) and (3.1), $F$ satisfies (2.8) and (2.9) with

$$
r=d\left(U_{1}, U_{2}\right), \quad \gamma=r^{2} / 4 \text { and } A=\left\|f_{1}\right\|_{2}\left\|f_{2}\right\|_{2}<\infty .
$$

Next, by assumption (4.21),

$$
|F(z)| \leq|\exp (-g(z))|\left\|f_{1}\right\|_{L^{1}\left(U_{1}, d \mu\right)}\left\|f_{2}\right\|_{L^{1}\left(U_{2}, d \mu\right)}=\exp (-\operatorname{Re} g(z)), \quad \forall z \in \mathcal{C}_{r^{2} / 4},
$$

that is, $F$ satisfies (2.15) with $B=1, \nu=0$. By Proposition 2.4.

$$
|F(z)| \leq \exp \left(-\operatorname{Re} g(z)+1-\operatorname{Re} \frac{r^{2}}{4 z}\right), \quad \forall z \in \mathcal{C}_{r^{2} / 4}
$$

Finally by Claim 1$]$

$$
\begin{aligned}
\sup _{x \in U_{1}} \sup _{x \in U_{2}}\left|p_{z}(x, y)\right| & =\sup \left\{\left\langle\exp (-z L) f_{1}, f_{2}\right\rangle:\left\|f_{1}\right\|_{L^{1}\left(U_{1}, d \mu\right)}=\left\|f_{2}\right\|_{L^{1}\left(U_{2}, d \mu\right)}=1\right\} \\
& \leq \exp \left(-\operatorname{Re} g(z)+1-\operatorname{Re} \frac{r^{2}}{4 z}\right)
\end{aligned}
$$

for all $z \in \mathcal{C}_{r^{2} / 4}$. The estimate for $z \notin \mathcal{C}_{r^{2} / 4}$ follows directly from (4.21).

\subsection{Operators acting on vector bundles.}

Our approach works not only for operators acting on functions but can also be applied to operators acting on vector bundles. To discuss the vector bundle version of our results we need some additional notation.

Let $(M, d, \mu)$ be a metric measure space and suppose that $T M$ is a continuous vector bundle with base $M$, fibers $T_{x} M \simeq \mathbb{C}^{n}$ and with continuous (with respect to $x$ ) scalar product $(\cdot, \cdot)_{x}$ on $T_{x} M$. For $f(x) \in T_{x} M$ we put $|f(x)|_{x}^{2}=(f(x), f(x))_{x}$. To simplify the notation, we will write $(\cdot, \cdot)$ and $|\cdot|$ instead of $(\cdot, \cdot)_{x}$ and $|\cdot|_{x}$. Now for sections $f$ and $g$ of $T M$ we put

$$
\|f\|_{L^{p}(M, d \mu ; T M)}^{p}=\int_{M}|f(x)|^{p} d \mu(x) \quad \text { and } \quad\langle f, g\rangle=\int_{M}(f(x), g(x)) d \mu(x) .
$$

Now let us describe the notion of integral operators for vector bundles. For any point $(x, y) \in M^{2}$ we consider the space $T_{y}^{*} \otimes T_{x}$. The space $T_{y}^{*} \otimes T_{x}$ is canonically isomorphic to $\operatorname{Hom}\left(T_{y}, T_{x}\right)$, the space of all linear homeomorphisms from $T_{y}$ to $T_{x}$. Denote again by $|\cdot|$ the operator norm on $T_{y}^{*} \otimes T_{x}$ with respect to the norms $|\cdot|_{x}$ and $|\cdot|_{y}$. 
By $\left(T^{*} \otimes T\right) M^{2}$ we denote the continuous bundle with base space equal to $M^{2}$ and with fiber over the point $(x, y)$ equal to $T_{y}^{*} \otimes T_{x}$. If there is a section $\vec{p}$ of $\left(T^{*} \otimes T\right) M^{2}$ such that $|\vec{p}|$ is a locally integrable function on $\left(M^{2}, \mu \times \mu\right)$ and $\vec{S} f_{1}$ is a section of $T M$ such that

$$
\left\langle\vec{S} f_{1}, f_{2}\right\rangle=\int_{M}\left(\vec{S} f_{1}(x), f_{2}(x)\right) d \mu(x)=\int_{M}\left(\vec{p}(x, y) f_{1}(y), f_{2}(x)\right) d \mu(y) d \mu(x)
$$

for all sections $f_{1}$ and $f_{2}$ in $C_{c}(T M)$, then we say that $\vec{S}$ is an integral operator on sections of $T M$ with kernel $\vec{p}$. As in the scalar case, $\vec{S}$ is a bounded linear operator from $L^{1}(M, d \mu ; T M)$ to $L^{\infty}(M, d \mu ; T M)$ if and only if $\vec{S}$ is an integral operator with kernel $\vec{p}$ such that $\operatorname{esssup}_{x, y \in M}|\vec{p}(x, y)|$ is finite, and

$$
\operatorname{esssup}_{x, y \in M}|\vec{p}(x, y)|=\|\vec{S}\|_{1 \rightarrow \infty} .
$$

One also has the following vector-valued version of Claim 11:

Claim 2. Let $U_{1}, U_{2}$ be open subsets of $M$. If $\vec{p}(x, y)$ is the kernel of a linear operator $\vec{S}: L^{1}(M, d \mu ; T M) \rightarrow L^{\infty}(M, d \mu ; T M)$, then

$$
\operatorname{esssup}_{x \in U_{1}, y \in U_{2}}|\vec{p}(x, y)|=\sup \left\{\left|\left\langle\vec{S} f_{1}, f_{2}\right\rangle\right|:\left\|f_{1}\right\|_{L^{1}\left(U_{1}, d \mu ; T M\right)}=\left\|f_{2}\right\|_{L^{1}\left(U_{2}, d \mu ; T M\right)}=1\right\} .
$$

Let us describe an example of Hodge type operator which generates a semigroup satisfying conditions (3.1) and (3.2) and acts on vector bundles of $k$-forms on Riemannian manifolds. Suppose that $M$ is a complete $n$-dimensional Riemannian manifold and $\mu$ is an absolutely continuous measure with a smooth density not equal to zero at any point of $M$. By $\Lambda^{k} T^{*} M$, $k=0, \ldots, n$, we denote the bundle of $k$-forms on $M$. For fixed $\beta, \beta^{\prime} \in L^{2}\left(\Lambda^{1} T^{*} M\right)$ and $\gamma \in L^{2}\left(\Lambda^{k} T^{*} M\right)$, we define the operator $\vec{L}=\vec{L}_{\beta, \beta^{\prime}, \gamma}$ acting on $L^{2}\left(\Lambda^{k} T^{*} M\right)$ by the formula

$$
\langle\vec{L} \omega, \omega\rangle=\int_{M}\left(\left|d_{k} \omega+\omega \wedge \beta\right|^{2}+\left|d_{n-k} * \omega+* \omega \wedge \beta^{\prime}\right|^{2}+|* \omega \wedge \gamma|^{2}\right) d \mu,
$$

where $\omega$ is a smooth compactly supported $k$-form and $*$ is the Hodge star operator. With some abuse of notation we also denote by $\vec{L}$ its Friedrichs extension. Note that for example the Hodge-Laplace operator and Schrödinger operators with real potentials and electromagnetic fields can be defined by (4.22). The following theorem was proved in [51].

Theorem 4.7. The self-adjoint semigroup $\left\{\exp (-z \vec{L}): z \in \mathbb{C}_{+}\right\}$generated by the operator $\vec{L}$ defined by (4.22) acts on $L^{2}\left(\Lambda^{k} T^{*} M\right)$ and satisfies (3.1) and (3.2).

Theorems 4.1, 4.2, 4.3, 4.6 and Corollary 4.4 can be extended to the above setting of operators acting on vector bundles. For example we can state Theorem 4.3 in this setting in the following way. Again, compare with [51, Corollary 9], which yields a slightly better estimate for real time and the Hodge-Laplace operator (and more generally operators defined by (4.22) ), but does not treat complex time. In what follows, Tr denotes the trace of an endomorphism on a finite dimensional linear space.

Theorem 4.8. Let $(M, d, \mu)$ be a metric measure space endowed with a vector bundle TM as above. Let $\vec{L}$ be a non-negative self-adjoint operator acting on $L^{2}(M, d \mu ; T M)$. Assume that $\vec{p}_{z}(x, y)$ is a continuous function of $x, y \in M$. Denote by $\vec{p}_{z}, z \in \mathbb{C}_{+}$, the kernel of $\exp (-z \vec{L})$. Let $V: \mathbb{R}_{+} \times M \rightarrow \mathbb{R}_{+}$be a continuous function satisfying condition (4.11). Assume that

$$
V(x, \sqrt{t}) \operatorname{Tr} \vec{p}_{t}(x, x) \leq 1, \quad \forall t>0, x \in M
$$


Then

$$
\left|\vec{p}_{z}(x, y)\right| \leq \frac{e K^{\prime}}{\sqrt{V\left(x, \frac{d(x, y)}{2}\right) V\left(y, \frac{d(x, y)}{2}\right)}}\left(\frac{d^{2}(x, y)}{4|z|}\right)^{\delta} \exp \left(-\operatorname{Re} \frac{d^{2}(x, y)}{4 z}\right)
$$

for all $z \in \mathbb{C}_{+}, x, y \in M$ such that $\operatorname{Re} \frac{d^{2}(x, y)}{4 z} \geq 1$.

Remark : Of course, one can transform the above estimate in a similar way as in Corollary 4.4.

Proof. Note that the self-adjointness of $\exp (-t \vec{L})$ implies $p_{t}(y, x)=\left[p_{t}(x, y)\right]^{*}$. Denote by |.| $\left.\right|_{H S}$ the Hilbert-Schmidt norm of a linear operator. One can write

$$
\begin{aligned}
\operatorname{Tr} \vec{p}_{t}(x, x) & =\operatorname{Tr} \int p_{t / 2}(x, y) p_{t / 2}(y, x) d \mu(y) \\
& =\operatorname{Tr} \int p_{t / 2}(x, y)\left[p_{t / 2}(x, y)\right]^{*} d \mu(y) \\
& =\int \operatorname{Tr}\left(p_{t / 2}(x, y)\left[p_{t / 2}(x, y)\right]^{*}\right) d \mu(y)
\end{aligned}
$$

thus

$$
\operatorname{Tr} \vec{p}_{t}(x, x)=\int\left|\vec{p}_{t / 2}(x, y)\right|_{H S}^{2} d \mu(y)
$$

On the other hand,

$$
\begin{aligned}
\left|\vec{p}_{t}(x, y)\right|^{2} & \leq \int\left|\vec{p}_{t / 2}(x, z)\right|^{2} d \mu(z) \int\left|\vec{p}_{t / 2}(z, y)\right|^{2} d \mu(z) \\
& \leq \int\left|\vec{p}_{t / 2}(x, z)\right|_{H S}^{2} d \mu(z) \int\left|\vec{p}_{t / 2}(z, y)\right|_{H S}^{2} d \mu(z)
\end{aligned}
$$

since $|.| \leq|\cdot|_{H S}$; hence, using (4.25) and (4.23),

$$
\left|\vec{p}_{t}(x, y)\right|^{2} \leq \frac{1}{V\left(x, t^{1 / 2}\right) V\left(y, t^{1 / 2}\right)}
$$

that is

$$
\left\|\mathbf{m}_{W(\cdot, \sqrt{t})} \exp (-t \vec{L}) \mathbf{m}_{W(\cdot, \sqrt{t})}\right\|_{1 \rightarrow \infty} \leq 1
$$

where $W(x, r)=\sqrt{V(x, r)}, x \in M, r>0$. This estimate can be extended to complex times. Indeed, one also has

$$
\int\left|\vec{p}_{t}(x, z)\right|^{2} d \mu(z) \leq \int\left|\vec{p}_{t}(x, z)\right|_{H S}^{2} d \mu(z) \leq \frac{1}{V\left(x, t^{1 / 2}\right)},
$$

that is

$$
\left\|\mathbf{m}_{W(\cdot, \sqrt{t})} \exp (-t \vec{L})\right\|_{2 \rightarrow \infty}=\left\|\exp (-t \vec{L}) \mathbf{m}_{W(\cdot, \sqrt{t})}\right\|_{1 \rightarrow 2} \leq 1, \quad \forall t>0
$$


Using the contractivity of $\exp (-i s \vec{L}), s \in \mathbb{R}$, on $L^{2}$, one has

$$
\begin{aligned}
& \left\|\mathbf{m}_{W(\cdot, \sqrt{\operatorname{Re} z})} \exp (-z \vec{L}) \mathbf{m}_{W(\cdot, \sqrt{\operatorname{Re} z})}\right\|_{1 \rightarrow \infty} \\
\leq & \left\|\mathbf{m}_{W(\cdot, \sqrt{\operatorname{Re} z})} \exp (-(z / 2) \vec{L})\right\|_{2 \rightarrow \infty}\left\|\exp (-(z / 2) \vec{L}) \mathbf{m}_{W(\cdot, \sqrt{\operatorname{Re} z})}\right\|_{1 \rightarrow 2} \\
\leq & \left\|\mathbf{m}_{W(\cdot, \sqrt{\operatorname{Re} z})} \exp (-(\operatorname{Re} z / 2) \vec{L})\right\|_{2 \rightarrow \infty}\left\|\exp (-(\operatorname{Re} z / 2) \vec{L}) \mathbf{m}_{W(\cdot, \sqrt{\operatorname{Re} z})}\right\|_{1 \rightarrow 2} \\
= & \left\|\exp (-(\operatorname{Re} z / 2) \vec{L}) \mathbf{m}_{W(\cdot, \sqrt{\operatorname{Re} z})}\right\|_{1 \rightarrow 2}^{2} .
\end{aligned}
$$

Together with (4.27) and the identity $\left\|T^{*} T\right\|_{1 \rightarrow \infty}=\|T\|_{1 \rightarrow 2}^{2}$, this yields

$$
\left\|\mathbf{m}_{W(\cdot, \sqrt{\operatorname{Re} z})} \exp (-z \vec{L}) \mathbf{m}_{W(\cdot, \sqrt{\operatorname{Re} z})}\right\|_{1 \rightarrow \infty} \leq 1, \forall z \in \mathbb{C}_{+} .
$$

Similarly as in (4.16), fix $x, y \in M$ and for $d(x, y)>2 s>0$ set $r=d(x, y)-2 s$. Consider the function $F$ defined by the formula

$$
F(z)=\left\langle\exp (-z \vec{L}) \mathbf{m}_{W\left(\cdot, \frac{r}{2}\right)} \omega_{2}, \mathbf{m}_{W\left(\cdot, \frac{r}{2}\right)} \omega_{1}\right\rangle,
$$

with $\omega_{1} \in L^{1}(B(x, s), d \mu ; T M) \cap L^{2}(B(x, s), V(\cdot, r / 2) d \mu ; T M), \omega_{2} \in L^{1}(B(y, s), d \mu ; T M) \cap$ $L^{2}(B(y, s), V(\cdot, r / 2) d \mu ; T M)$, and $\left\|\omega_{1}\right\|_{1}=\left\|\omega_{2}\right\|_{1}=1$. In virtue of assumption (3.1) and Davies-Gaffney estimates (3.2), $F$ satisfies (2.8) and (2.9) with $\gamma=r^{2} / 4$ and

$$
A=\left\|\mathbf{m}_{W\left(\cdot, \frac{r}{2}\right)} \omega_{1}\right\|_{2}\left\|\mathbf{m}_{W\left(\cdot, \frac{r}{2}\right)} \omega_{2}\right\|_{2}=\left\|\omega_{1}\right\|_{L^{2}\left(U_{1}, V(\cdot, r / 2) d \mu\right)}\left\|\omega_{2}\right\|_{L^{2}\left(U_{2}, V(\cdot, r / 2) d \mu\right)} .
$$

Now if $z \in \mathcal{C}_{r^{2} / 4}$, then $\sqrt{\operatorname{Re} z} \leq r / 2$ by (4.17). Using the assumptions on $\omega_{1}, \omega_{2}, W$ as well as (4.28), we obtain

$$
\begin{aligned}
|F(z)| & =\left|\left\langle\mathbf{m}_{W\left(\cdot, \frac{r}{2}\right)} \exp (-z \vec{L}) \mathbf{m}_{W\left(\cdot, \frac{r}{2}\right)} \omega_{2}, \omega_{1}\right\rangle\right| \\
& \leq \sup _{x, y \in M} \frac{W\left(x, \frac{r}{2}\right) W\left(y, \frac{r}{2}\right)}{W(x, \sqrt{\operatorname{Re} z}) W(y, \sqrt{\operatorname{Re} z})}\left|\left\langle\mathbf{m}_{W(\cdot, \sqrt{\operatorname{Re} z})} \exp (-z \vec{L}) \mathbf{m}_{W(\cdot, \sqrt{\operatorname{Re} z})} \omega_{2}, \omega_{1}\right\rangle\right| \\
& \leq \sup _{x, y \in M} \frac{W\left(x, \frac{r}{2}\right) W\left(y, \frac{r}{2}\right)}{W(x, \sqrt{\operatorname{Re} z}) W(y, \sqrt{\operatorname{Re} z})}\left\|\mathbf{m}_{W(\cdot, \sqrt{\operatorname{Re} z})} \exp (-z \vec{L}) \mathbf{m}_{W(\cdot, \sqrt{\operatorname{Re} z})}\right\|_{1 \rightarrow \infty} \\
& \leq K^{\prime}\left(\frac{r^{2}}{4 \operatorname{Re} z}\right)^{\delta / 2}
\end{aligned}
$$

for all $z \in \mathcal{C}_{r^{2} / 4}$. Thus $F$ satisfies (2.10) with $B=K^{\prime} 2^{-\delta}$ and $\nu=\delta$. By Proposition 2.3.

$$
|F(z)| \leq e K^{\prime}\left(\frac{r^{2}}{4|z|}\right)^{\delta} \exp \left(-\operatorname{Re} \frac{r^{2}}{4 z},\right)
$$

for all $z \in \mathcal{C}_{r^{2} / 4}$. One finishes the proof as in Theorem 4.3, using Claim 2 ,

4.4. Gaussian estimates for the gradient of the heat kernel. The technique which we developed above can be applied to obtain Gaussian bounds for gradient of the heat kernels. The following result is motivated by some considerations in [4, Section 1.4. In particular, it is proved in 4 that under the assumptions below, for $a=1 / 2$, the Riesz transform is bounded on $L^{p}(M, d \mu)$ for $2<p<+\infty$. In the langage of [4], we will show now that, under $(F K)$, conditions (1.7), (1.8) and $(G)$ are all equivalent, which was left open there. A similar result was obtained independently in [30, by a different method, relying directly on the finite speed propagation property for the wave equation. 
Theorem 4.9. Let $M$ be a complete Riemannian manifold such that the Riemannian measure $\mu$ satisfies the doubling condition (4.9) and let $\Delta$ be the Laplace-Beltrami operator, $p_{z}$ the corresponding heat kernel, $\nabla$ the Riemannian gradient on $M$. Suppose next that

$$
p_{t}(x, x) \leq \frac{C}{\mu(B(x, \sqrt{t}))}, \forall t>0, x \in M,
$$

and that

$$
\sup _{x, y \in M}\left|\nabla p_{t}(x, y)\right| \mu(B(y, \sqrt{t})) \leq C t^{-a}
$$

for some $a>0$ and all $t \in \mathbb{R}_{+}$. Then

$$
\left|\nabla p_{t}(x, y)\right| \leq \frac{C}{t^{a} \mu(B(y, \sqrt{t}))}\left(1+\frac{d^{2}(x, y)}{4 t}\right)^{3 \delta+2 a} \exp \left(-\frac{d^{2}(x, y)}{4 t}\right)
$$

for all $t>0, x, y \in M$.

In the proof of Theorem 4.9 we shall need the following consequence of Corollary 4.4 and assumption.

Lemma 4.10. Assume (4.9), (4.29), and (4.30). Then

$$
\left\|\nabla \exp (-z \Delta) \mathbf{m}_{V(\cdot, r)}\right\|_{1 \rightarrow \infty}=\sup _{x, y \in M}\left|\nabla p_{z}(x, y)\right| V(y, r) \leq C(\operatorname{Re} z)^{-a}\left(\frac{|z|}{\operatorname{Re} z}\right)^{2 \delta}\left(\frac{r^{2}}{\operatorname{Re} z}\right)^{\delta / 2}
$$

for all $r>0, z \in \mathcal{C}_{r^{2} / 4}$, where $\delta>0$ is the exponent in (4.10).

Proof. An immediate reformulation of (4.18) is

$$
\left|p_{z}(x, y)\right| \leq \frac{e K^{\prime}\left(1+\operatorname{Re} \frac{d^{2}(x, y)}{4 z}\right)^{\delta}}{\sqrt{V\left(x,\left(\operatorname{Re} \frac{1}{z}\right)^{-1 / 2}\right) V\left(y,\left(\operatorname{Re} \frac{1}{z}\right)^{-1 / 2}\right)}} \exp \left(-\operatorname{Re} \frac{d^{2}(x, y)}{4 z}\right)\left(\frac{|z|}{\operatorname{Re} z}\right)^{\delta}
$$

which yields, for $0<c<1 / 4$,

$$
\left|p_{z}(x, y)\right| \leq \frac{C}{\sqrt{V\left(x,\left(\operatorname{Re} \frac{1}{z}\right)^{-1 / 2}\right) V\left(y,\left(\operatorname{Re} \frac{1}{z}\right)^{-1 / 2}\right)}} \exp \left(-c \operatorname{Re} \frac{d^{2}(x, y)}{z}\right)\left(\frac{|z|}{\operatorname{Re} z}\right)^{\delta}
$$

and, by doubling,

$$
\left|p_{z}(x, y)\right| \leq \frac{C^{\prime}}{V\left(y,\left(\operatorname{Re} \frac{1}{z}\right)^{-1 / 2}\right)} \exp \left(-c^{\prime} \operatorname{Re} \frac{d^{2}(x, y)}{z}\right)\left(\frac{|z|}{\operatorname{Re} z}\right)^{\delta}
$$

Take now $z=t+i s \in \mathcal{C}_{r^{2} / 4}$, where $t, s \in \mathbb{R}$. Note that $(t / 2)+i s \in \mathcal{C}_{r^{2}}$. Write

$$
\nabla p_{z}(x, y)=\int_{M} \nabla p_{t / 2}(x, u) p_{(t / 2)+i s}(u, y) d \mu(u)
$$

hence, using (4.4), (4.30), and doubling,

$$
\left|\nabla p_{z}(x, y)\right| \leq \frac{C}{t^{a} V\left(y,\left(\operatorname{Re} \frac{1}{z}\right)^{-1 / 2}\right)}\left(\frac{|z|}{\operatorname{Re} z}\right)^{\delta} \int_{M} \frac{1}{V(u, \sqrt{t})} \exp \left(-c^{\prime} \operatorname{Re} \frac{d^{2}(u, y)}{z}\right) d \mu(u) .
$$


Let us estimate

$$
I=\int_{M} \frac{1}{V(u, \sqrt{t})} \exp \left(-c^{\prime} \operatorname{Re} \frac{d^{2}(u, y)}{z}\right) d \mu(u) .
$$

Since $\left(\operatorname{Re} \frac{1}{z}\right)^{-1 / 2} \geq(\operatorname{Re} z)^{1 / 2}=\sqrt{t}$, one has

$$
I \leq K^{\prime}\left(\frac{\left(\operatorname{Re} \frac{1}{z}\right)^{-1 / 2}}{\sqrt{\operatorname{Re} z}}\right)^{\delta / 2} I I=\left(\frac{|z|}{\operatorname{Re} z}\right)^{\delta} I I,
$$

where

$$
I I=\int_{M} \frac{1}{V\left(u,\left(\operatorname{Re} \frac{1}{z}\right)^{-1 / 2}\right)} \exp \left(-c^{\prime} \operatorname{Re} \frac{d^{2}(u, y)}{z}\right) d \mu(u)
$$

is easily seen to be uniformly bounded in $y \in M, z \in \mathbb{C}_{+}$by doubling. Thus

$$
\left|\nabla p_{z}(x, y)\right| \leq \frac{C}{t^{a} V\left(y,\left(\operatorname{Re} \frac{1}{z}\right)^{-1 / 2}\right)}\left(\frac{|z|}{\operatorname{Re} z}\right)^{2 \delta} .
$$

Now, for $z \in \mathcal{C}_{r^{2}}, r \geq\left(\operatorname{Re} \frac{1}{z}\right)^{-1 / 2} \geq(\operatorname{Re} z)^{1 / 2}$, hence

$$
\frac{V(y, r)}{V\left(y,\left(\operatorname{Re} \frac{1}{z}\right)^{-1 / 2}\right)} \leq \frac{V(y, r)}{V\left(y,(\operatorname{Re} z)^{1 / 2}\right)} \leq K^{\prime}\left(\frac{r^{2}}{\operatorname{Re} z}\right)^{\delta / 2},
$$

therefore

$$
\left|\nabla p_{z}(x, y)\right| V(y, r) \leq C(\operatorname{Re} z)^{-a}\left(\frac{r^{2}}{\operatorname{Re} z}\right)^{\delta / 2}\left(\frac{|z|}{\operatorname{Re} z}\right)^{2 \delta},
$$

which is the claim.

Proof of Theorem 4.9. As before fix $x, y \in M$ and, for $0<2 s<d(x, y)$, put $r=d(x, y)-2 s$. Next fix $f \in L^{2}(B(x, s), d \mu) \cap L^{1}(B(x, s), d \mu)$ and let $X \in T M$ be a smooth vector field on $M$ supported in $B(y, s)$. This time we set, for $z \in \mathbb{C}_{+}$,

$$
F(z)=\left\langle\nabla \exp (-z \Delta) \mathbf{m}_{V(\cdot, r)} f, X\right\rangle,
$$

where $V(x, r)=\mu(B(x, r))$. Now

$$
F(z)=\left\langle\exp (-z \Delta) \mathbf{m}_{V(\cdot, r)} f, \nabla^{*} X\right\rangle=\left\langle\exp (-z \Delta) \mathbf{m}_{V(\cdot, r)} f, \operatorname{div} X\right\rangle,
$$

Therefore, since $\Psi(z)=e^{-z \Delta}$ satisfies (3.1) and (3.2), $F$ satisfies (2.8) and (2.9) with

$$
A=\left\|\mathbf{m}_{V(\cdot, r)} f\right\|_{2}\|\operatorname{div} X\|_{2} \text { and } \gamma=r^{2} / 4 \text {. }
$$

Note that $A$ is finite since $\operatorname{div} X$ is smooth and supported in $B(y, s)$. Now assume in addition that $\|f\|_{1}=\|X \mid\|_{1}=1$ and let $z \in \mathcal{C}_{r^{2} / 4}$. By Lemma 4.10.

$$
\begin{aligned}
|F(z)| & \leq\left\|\nabla \exp (-z \Delta) \mathbf{m}_{V(\cdot, r)}\right\|_{1 \rightarrow \infty} \\
& \leq C(\operatorname{Re} z)^{-a}\left(\frac{|z|}{\operatorname{Re} z}\right)^{2 \delta}\left(\frac{r^{2}}{\operatorname{Re} z}\right)^{\delta / 2}=C r^{-2 a-4 \delta}\left(\frac{r^{2}}{\operatorname{Re} z}\right)^{(5 \delta / 2)+a}|z|^{2 \delta}
\end{aligned}
$$

for all $z \in \mathcal{C}_{r^{2} / 4}$. Thus $F$ satisfies the assumptions of Proposition 2.4 with

$$
B=C r^{-2 a-4 \delta}, \quad \nu=5 \delta+2 a, \quad \gamma=r^{2} / 4, \quad \exp (g(z))=z^{-2 \delta}, \quad \text { and any } \beta \in(0,1) .
$$


Therefore

$$
|F(z)| \leq e C r^{-2 a-4 \delta}\left(\frac{r^{2}}{2|z|}\right)^{5 \delta+2 a}|z|^{2 \delta} \exp \left(-\operatorname{Re} \frac{r^{2}}{4 z}\right)
$$

for all $z \in \mathcal{C}_{r^{2} / 4}$. An obvious modification of Claim 2 yields

$$
\begin{aligned}
\left|\nabla p_{z}(x, y)\right| V(y, r) & \leq \sup _{x^{\prime} \in B(x, s)} \sup _{y^{\prime} \in B(y, s)}\left|\nabla p_{z}\left(x^{\prime}, y^{\prime}\right)\right| V\left(y^{\prime}, r\right) \\
& =\sup \left\{|F(z)|:\|f\|_{L^{1}(B(x, s), d \mu)}=\|X\|_{L^{1}(B(y, s), d \mu)}=1\right\} \\
& \leq C r^{-2 a}\left(\frac{r^{2}}{4|z|}\right)^{3 \delta+2 a} \exp \left(-\operatorname{Re} \frac{r^{2}}{4 z}\right),
\end{aligned}
$$

and letting $s$ go to 0 we obtain

$$
\left|\nabla p_{z}(x, y)\right| V(y, d(x, y)) \leq C\left(d^{2}(x, y)\right)^{-a}\left(\frac{d^{2}(x, y)}{4|z|}\right)^{3 \delta+2 a} \exp \left(-\operatorname{Re} \frac{d^{2}(x, y)}{4 z}\right)
$$

for all $z \in \mathcal{C}_{d^{2}(x, y) / 4}$. If $0 \leq t \leq d^{2}(x, y) / 4$ then $V(y, \sqrt{t}) \leq V\left(y, \frac{d(x, y)}{2}\right)$, so by (4.33)

$$
\left|\nabla p_{t}(x, y)\right| V(y, \sqrt{t}) \leq C t^{-a}\left(\frac{d^{2}(x, y)}{4 t}\right)^{3 \delta+2 a} \exp \left(-\frac{d^{2}(x, y)}{4 t}\right)
$$

for all $0 \leq t \leq d^{2}(x, y) / 4$. For $t \geq d^{2}(x, y) / 4$, (4.31) is immediate from (4.30).

Remark: Note that (4.33) also yields complex time estimates for the gradient of the heat kernel.

4.5. Families of operators without semigroup property. An important advantage of the technique which we discuss here is that we do not have to assume that the family $\left\{\Psi(z): z \in \mathbb{C}_{+}\right\}$under consideration has the semigroup property. Hence we are able to apply our results to families operators which can be defined by: $\Psi(z)=g(z) \exp (-z L)$, where $g: \mathbb{C}_{+} \rightarrow \mathbb{C}$ is an analytic function; $\Psi(z)=\exp \left(-z L_{1}\right) \exp \left(-z L_{2}\right) ; \Psi(z)=\exp \left(-z L_{1}\right)-$ $\exp \left(-z L_{2}\right)$ or some more complex formulae. To be more precise, let us come back to the general metric measure space setting, and consider an analytic family of operators $\left\{\Psi(z): z \in \mathbb{C}_{+}\right\}$acting on $L^{2}(M, d \mu)$. Next assume that (see (4.1))

$$
\|\Psi(z)\|_{1 \rightarrow \infty} \leq K(\operatorname{Re} z)^{-D / 2}, \quad \forall z \in \mathbb{C}_{+} .
$$

By [29, Theorem 6, p.503] we can define the kernel $p_{z}^{\Psi}$ of the operator $\Psi(z)$ in the same way as in (4.2) and again assume that $p_{z}^{\Psi}$ is a continuous function on $M^{2}$. Now we can state the following version of Theorem 4.1 .

Theorem 4.11. Suppose that the family $\left\{\Psi(z): z \in \mathbb{C}_{+}\right\}$satisfies conditions (3.1), (3.2) and (4.34). Then the kernel $p_{z}^{\Psi}$, if continuous, satisfies estimates (4.5).

The proof of Theorem 4.11 is the same as the proof of Theorem 4.1 .

For instance, let $L$ be a self-adjoint uniform elliptic second order differential operator in divergence form with periodic coefficients acting on $L^{2}\left(\mathbb{R}^{n}\right)$, and let $L^{o}$ be the corresponding homogenized operator. Next let $p_{z}$ and $p_{z}^{o}$ be the corresponding heat kernels. Then a 
straightforward modification of the argument from [57 shows the following so-called BerryEsseen type estimate

$$
\left|p_{z}(x, y)-p_{z}^{o}(x, y)\right| \leq K \min \left\{1, \frac{|z|}{(\operatorname{Re} z)^{3 / 2}}\right\}(\operatorname{Re} z)^{-n / 2}
$$

for all $z \in \mathbb{C}_{+}, x, y \in \mathbb{R}^{n}$. The following consequence of Theorem 4.11 can be used to obtain Gaussian bounds for the expression $\left|p_{z}(x, y)-p_{z}^{o}(x, y)\right|$.

Example 4.12. Suppose that $L$ and $L^{0}$ are two generators of analytic semigroups on $L^{2}\left(\mathbb{R}^{n}\right)$. Next assume that $L$ and $L^{0}$ satisfy conditions (3.1), (3.2) with the distances $d$ and $d^{0}$. Finally suppose that the corresponding heat kernels satisfy estimate (4.35) and set $\tilde{d}(x, y)=$ $\min \left\{d(x, y), d^{0}(x, y)\right\}$. Then

$$
\left|p_{z}(x, y)-p_{z}^{0}(x, y)\right| \leq \frac{e K \min \left\{1, \frac{|z|}{(\operatorname{Re} z)^{3 / 2}}\right\}}{(\operatorname{Re} z)^{n / 2}}\left(1+\operatorname{Re} \frac{\tilde{d}^{2}(x, y)}{4 z}\right)^{\frac{n+3}{2}} \exp \left(-\operatorname{Re} \frac{\tilde{d}^{2}(x, y)}{4 z}\right)
$$

for all $z \in \mathbb{C}_{+}, x, y \in \mathbb{R}^{n}$.

Proof. It easy to note that if for $z \in \mathbb{C}_{+}$we put $\Psi(z)=\exp (-z L)-\exp \left(-z L^{0}\right)$ then $\Psi$ satisfies (3.1) and (3.2) with the distance $\tilde{d}$ (and with constant 2). Hence Example 4.12 follows from Proposition 2.4 with $\exp (g(z))=z$ and $\nu=n+3$. Note that with this choice of $g$, (2.14) is satisfied for any $\beta>0$, for some constants $C$ and $c$ depending on $\gamma$ and $\beta$.

Remark : As we already said, the fact that the exponent in the polynomial correction factor in front of the exponential in (4.5) cannot be improved to $(D-1) / 2$ as in [49] is related to the fact that the proof of Theorem 4.1 does not use the fact that the family of operators under consideration is a semigroup, in other words it is related to the possibility of extending Theorem 4.1 to Theorem 4.11. Indeed, let $L$ be the standard Laplace operator acting on $\mathbb{R}^{n}$, and apply Theorem 4.11 to the family of operators $\Psi(z)=z^{n / 2} \exp (-z L)$, $z \in \mathbb{C}_{+}$. In this case $D=0$ in (4.34), and the conclusion cannot hold with $(D-1) / 2=-1 / 2$ in the polynomial correction factor in front of the exponential, since in that case $p_{z}(x, y)$ is exactly given by the Gauss function. By contrast, the argument from 49] cannot be applied to this choice of $\Psi(z)$, because it only applies to semigroups.

A more elementary example is the following. Let $M=\{x, y\}$ with counting measure and let $d(x, y)=1$. Consider the analytic family of operators $\left\{\Psi(z): z \in \mathbb{C}_{+}\right\}$acting on $L^{2}(M)$ given by the kernel $p_{z}(x, x)=p_{z}(y, y)=0$ and $p_{z}(x, y)=p_{z}(y, x)=\exp \left(-\frac{1}{4 z}\right)$. It is easy to check that the family $\left\{\Psi(z): z \in \mathbb{C}_{+}\right\}$satisfies conditions (3.1), (3.2) and (4.34) with $D=0$. Again, this shows that the exponent $D / 2$ cannot be replaced by $(D-1) / 2$ in the setting of Theorem 4.11.

4.6. $L^{p} \rightarrow L^{q}$ Gaussian estimates. Claim 1 reduces the proof of Gaussian bounds for the heat kernel to obtaining a Gaussian type estimate for expressions of the form

$$
\sup \left\{\left|\left\langle\exp (-z L) f_{1}, f_{2}\right\rangle\right|:\left\|f_{1}\right\|_{L^{1}\left(U_{1}, d \mu\right)}=\left\|f_{2}\right\|_{L^{1}\left(U_{2}, d \mu\right)}=1\right\} .
$$

In such expressions, one can replace the $L^{1}$ norms of functions $f_{1}$ and $f_{2}$ by the $L^{p}$ norm of $f_{1}$ and the $L^{q}$ norm of $f_{2}$, for $1 \leq p, q \leq+\infty$. This leads to natural generalizations of pointwise Gaussian bounds and provides some form of Gaussian bounds for semigroups without heat kernels. There are many interesting examples of operators which generate such semigroups. More precisely, the corresponding semigroup $\exp (-t L)$ is not bounded from 
$L^{1}$ to $L^{\infty}$ even locally. The kernel of the operator $\exp (-t L)$ can always be defined as a distribution or in some other sense, but in such cases it is not a bounded function. Often such operators generate bounded semigroups on $L^{p}$ spaces only for $p$ ranging in some proper subinterval of $[1, \infty]$. We discuss a semigroup of this type in Example 4.15 below.

The so-called generalized Gaussian bounds that such semigroups may satisfy were studied for instance by Davies in [25, Lemmas 23 and 24], and they were extensively discussed by Blunck and Kunstmann (see [8, 9, 10, 11, 12). Estimates of a similar nature were also considered in [42], see Propositions 2.6 and 2.8 in this paper. For interesting considerations about $L^{p}-L^{q}$ Gaussian estimates, see also [5].

As in Section 4.5. we consider analytic families of operators $\left\{\Psi(z): z \in \mathbb{C}_{+}\right\}$on metric measure spaces rather than semigroups generated by self-adjoint operators. Still the case $\Psi(z)=\exp (-z L)$ is the most natural example. In this section we are going to consider families of operators satisfying the following condition

$$
\left\|\mathbf{m}_{W_{1}(\cdot, \sqrt{\operatorname{Re} z})} \Psi(z) \mathbf{m}_{W_{2}(\cdot, \sqrt{\operatorname{Re} z})}\right\|_{p \rightarrow q} \leq 1, \quad \forall z \in \mathbb{C}_{+},
$$

where $1 \leq p<q \leq+\infty$, and the functions $W_{i}$ satisfy condition (4.11) with exponents $\delta_{i} / 2$ for $i=1,2$. We discuss the rationale for condition (4.36) in remark (b) after Theorem 4.13, Now let us notice only that if $\Psi(z)=\exp (-z L), p=1, q=\infty$ and $W_{1}=W_{2}=V^{1 / 2}$, then estimates (4.36) are equivalent to estimates (4.14), which follow as we have seen from condition (4.12). Hence one can think of condition (4.36) as a generalization of the ondiagonal estimates (4.12).

Theorem 4.13. Let $(M, d, \mu)$ be a metric measure space. Suppose that the functions $W_{i}: \mathbb{R}_{+} \times M \rightarrow \mathbb{R}_{+}, i=1,2$, are continuous and satisfy condition (4.11) with constants $\sqrt{K_{i}^{\prime}} \geq 1$ and exponents $\delta_{i} / 2$. Next assume that the analytic family $\left\{\Psi(z): z \in \mathbb{C}_{+}\right\}$ of operators on $L^{2}(M, d \mu)$ satisfies conditions (3.1), (3.2) as well as (4.36) for some $p, q$, $1 \leq p<q \leq+\infty$. Then

$$
\left\|P_{1} \mathbf{m}_{W_{1}\left(\cdot, \frac{r}{2}\right)} \Psi(z) \mathbf{m}_{W_{2}\left(\cdot, \frac{r}{2}\right)} P_{2}\right\|_{p \rightarrow q} \leq e \sqrt{K_{1}^{\prime} K_{2}^{\prime}}\left(\frac{r^{2}}{4|z|}\right)^{\delta_{1}+\delta_{2}} \exp \left(-\operatorname{Re} \frac{r^{2}}{4 z}\right)
$$

for all $U_{1}, U_{2}$ open subsets of $M$ and all $z \in \mathcal{C}_{r^{2} / 4}$, with $r=d\left(U_{1}, U_{2}\right)$. Here $P_{i}$ denotes the operator of multiplication by the characteristic function of the sets $U_{i} \subset M$, that is $P_{i}=\mathbf{m}_{\chi_{U_{i}}}$ for $i=1,2$. Moreover

$$
\left\|P_{1} \mathbf{m}_{W_{1}(\cdot, \sqrt{t})} \Psi(t) \mathbf{m}_{W_{2}(\cdot, \sqrt{t})} P_{2}\right\|_{p \rightarrow q} \leq e \sqrt{K_{1}^{\prime} K_{2}^{\prime}}\left(1+\frac{r^{2}}{4 t}\right)^{\delta_{1}+\delta_{2}} \exp \left(-\frac{r^{2}}{4 t}\right)
$$

for all $t \in \mathbb{R}_{+}$.

Proof. Similarly as in (4.16) we consider the function $F$ defined by the formula

$$
F(z)=\left\langle\Psi(z) \mathbf{m}_{W_{2}\left(\cdot, \frac{r}{2}\right)} f_{2}, \mathbf{m}_{W_{1}\left(\cdot, \frac{r}{2}\right)} f_{1}\right\rangle
$$

but now assume that $f_{i} \in L^{2}\left(U_{i}, W_{i}^{2}(\cdot, r / 2) d \mu\right)$ for $i=1,2$ and $\left\|f_{1}\right\|_{L^{q^{\prime}}\left(U_{1}, d \mu\right)}=\left\|f_{2}\right\|_{L^{p}\left(U_{2}, d \mu\right)}=$ 1 , where $1 / q+1 / q^{\prime}=1$.

In virtue of assumption (3.1) and Davies-Gaffney estimates (3.2), $F$ satisfies (2.8) and (2.9) with $\gamma=r^{2} / 4$ and

$$
A=\left\|\mathbf{m}_{W_{1}\left(\cdot, \frac{r}{2}\right)} f_{1}\right\|_{2}\left\|\mathbf{m}_{W_{2}\left(\cdot, \frac{r}{2}\right)} f_{2}\right\|_{2}=\left\|f_{1}\right\|_{L^{2}\left(U_{1}, W_{1}^{2}(\cdot, r / 2) d \mu\right)}\left\|f_{2}\right\|_{L^{2}\left(U_{2}, W_{2}^{2}(\cdot, r / 2) d \mu\right)} .
$$


Now if $z \in \mathcal{C}_{r^{2} / 4}$, then $\sqrt{\operatorname{Re} z} \leq r / 2$ by (4.17). Using the assumptions on $f_{1}, f_{2}, W_{1}, W_{2}$ as well as (4.36), we obtain

$$
\begin{aligned}
|F(z)| & =\left|\left\langle\mathbf{m}_{W_{1}\left(\cdot, \frac{r}{2}\right)} \Psi(z) \mathbf{m}_{W_{2}\left(\cdot, \frac{r}{2}\right)} f_{2}, f_{1}\right\rangle\right| \leq\left\|\mathbf{m}_{W_{1}\left(\cdot, \frac{r}{2}\right)} \Psi(z) \mathbf{m}_{W_{2}\left(\cdot, \frac{r}{2}\right)}\right\|_{p \rightarrow q} \\
& \leq \sup _{x, y \in M} \frac{W_{1}\left(x, \frac{r}{2}\right) W_{2}\left(y, \frac{r}{2}\right)}{W_{1}(x, \sqrt{\operatorname{Re} z}) W_{2}(y, \sqrt{\operatorname{Re} z})}\left\|\mathbf{m}_{W_{1}(\cdot, \sqrt{\operatorname{Re} z})} \Psi(z) \mathbf{m}_{W_{2}(\cdot, \sqrt{\operatorname{Re} z})}\right\|_{p \rightarrow q} \\
& \leq \sqrt{K_{1}^{\prime} K_{2}^{\prime}}\left(\frac{r^{2}}{4 \operatorname{Re} z}\right)^{\left(\delta_{1}+\delta_{2}\right) / 2}=\sqrt{K_{1}^{\prime} K_{2}^{\prime}} 2^{-\left(\delta_{1}+\delta_{2}\right)}\left(\frac{\operatorname{Re} z}{r^{2}}\right)^{-\left(\delta_{1}+\delta_{2}\right) / 2}
\end{aligned}
$$

for all $z \in \mathcal{C}_{r^{2} / 4}$. Thus $F$ satisfies (2.10) with $B=\sqrt{K_{1}^{\prime} K_{2}^{\prime}} 2^{-\left(\delta_{1}+\delta_{2}\right)}$ and $\nu=\delta_{1}+\delta_{2}$. By Proposition 2.3 .

$$
|F(z)| \leq e \sqrt{K_{1}^{\prime} K_{2}^{\prime}} 2^{-\left(\delta_{1}+\delta_{2}\right)}\left(\frac{r^{2}}{2|z|}\right)^{\delta_{1}+\delta_{2}} \exp \left(-\operatorname{Re} \frac{r^{2}}{4 z}\right) .
$$

The spaces $L^{1}\left(U_{i}, d \mu\right) \cap L^{2}\left(U_{i}, W_{i}^{2}(\cdot, r / 2) d \mu\right), i=1,2$, being dense in $L^{q^{\prime}}\left(U_{1}, d \mu\right)$ (resp. $\left.L^{p}\left(U_{2}, d \mu\right)\right)$, the above inequality, for all functions $f_{i} \in L^{2}\left(U_{i}, W_{i}^{2}(., r / 2) d \mu\right), i=1,2$, such that $\left\|f_{1}\right\|_{q^{\prime}}=\left\|f_{2}\right\|_{p}=1$, yields (4.37).

To prove (4.38) we notice that for $t \leq r^{2} / 4$, it is a straightforward consequence of (4.37). For $t \geq r^{2} / 4$, it follows from (4.36).

Remarks : (a) Note that if $\Psi(z)=\exp (-z L), 1 / p+1 / p^{\prime}=1$, and $W_{1}=W_{2}=W$, then it is enough to assume that (4.36) holds for $z=t \in \mathbb{R}_{+}$. Indeed, by using, as in the proof of Theorem 4.8, the identity $\left\|T^{*} T\right\|_{p \rightarrow p^{\prime}}=\|T\|_{p \rightarrow 2}^{2}$ and the contractivity of $\exp (-i s L), s \in \mathbb{R}$, on $L^{2}(M, d \mu)$, one obtains

$$
\left\|\mathbf{m}_{W(\cdot, \sqrt{\operatorname{Re} z})} \exp (-z L) \mathbf{m}_{W(\cdot, \sqrt{\operatorname{Re} z})}\right\|_{p \rightarrow p^{\prime}}=\left\|\mathbf{m}_{W(\cdot, \sqrt{\operatorname{Re} z})} \exp (-(\operatorname{Re} z) L) \mathbf{m}_{W(\cdot, \sqrt{\operatorname{Re} z})}\right\|_{p \rightarrow p^{\prime}} .
$$

(b) In [8, 9, 10, 11, 12, Blunck and Kunstmann develop spectral multiplier theorems for operators which generate semigroups without heat kernel acting on spaces satisfying the doubling condition ${ }^{4}$. As their basic assumption they consider the following form of generalized Gaussian estimates

$$
\left\|\mathbf{m}_{\chi_{B(x, \sqrt{t})}} \exp (-t L) \mathbf{m}_{\chi_{B(y, \sqrt{t})}}\right\|_{p \rightarrow q} \leq C V(x, \sqrt{t})^{\frac{1}{q}-\frac{1}{p}} \exp \left(-c \frac{d^{2}(x, y)}{t}\right), \forall t>0, x, y \in M,
$$

where $1 \leq p \leq 2 \leq q \leq+\infty$ and $V(x, r)=\mu(B(x, r))$. The above estimates imply that (4.40) $\left\|\exp (-t L) \mathbf{m}_{V^{(1 / 2)-(1 / p)}(\cdot, \sqrt{t})}\right\|_{p \rightarrow 2} \leq C \quad$ and $\quad\left\|\mathbf{m}_{V^{(1 / q)-(1 / 2)}(\cdot, \sqrt{t})} \exp (-t L)\right\|_{2 \rightarrow q} \leq C$ (see [12, Proposition 2.1, (ii)]). It follows from the above considerations that the opposite implication is valid if $(M, d, \mu, L)$ satisfies conditions (3.1) and (3.2). Indeed, estimates (4.40) imply (4.36) with $W_{1}=V^{(1 / 2)-(1 / q)}(\cdot, \sqrt{t})$ and $W_{2}=V^{(1 / p)-(1 / 2)}(\cdot, \sqrt{t})$. Now, according to Theorem 4.13, (4.40) implies the estimates (4.38), which in turn imply (4.39) by choosing $U_{1}=B(x, \sqrt{t}), U_{2}=B(y, \sqrt{t})$ and using doubling. Thus Theorem 4.13 can be used to verify the main assumption of the results obtained in [8, 19, 10, 11, 12. For example the next statement follows from [9, Theorem 1.1] and Theorem 4.13 (see also [14, Theorem 4.3],

\footnotetext{
${ }^{4}$ Here we discuss only second-order operators. Blunck and Kunstmann consider also the mth-order version of generalized Gaussian estimates.
} 
for a more primitive version, with an additional $\varepsilon$ in the resulting exponent). We give below a proof that follows directly from Theorem 4.13. The conclusion of the corollary is instrumental in the theory of Riesz means (see [14, 9], and references therein); see also condition $\left(H G_{\alpha}\right)$, p.339 in 32 and its consequences.

Corollary 4.14. Suppose that $(M, d, \mu, L)$ satisfies the doubling condition (4.9), as well as conditions (3.1) and (3.2). Let $p \in[1,2]$, and assume that there exists $C>0$ such that

$$
\left\|\exp (-t L) \mathbf{m}_{V^{(1 / p)-(1 / 2)}(\cdot, \sqrt{t})}\right\|_{p \rightarrow 2} \leq C, \quad \forall t \in \mathbb{R}_{+},
$$

where $V(x, r)=\mu(B(x, r))$. Then there exists $C>0$ such that

$$
\|\exp (-z L)\|_{\tilde{p} \rightarrow \tilde{p}} \leq C\left(\frac{|z|}{\operatorname{Re} z}\right)^{\delta \mid \frac{1}{\tilde{p}-\frac{1}{2} \mid}}, \forall z \in \mathbb{C}_{+}, \tilde{p} \in\left[p, p^{\prime}\right]
$$

where $\delta$ is the exponent in condition (4.10).

Proof. Note that, for all $p \in[1, \infty]$,

$$
\sum_{k}\left(\sum_{l}\left|c_{l k} a_{l}\right|\right)^{p} \leq\left(\max \left\{\sup _{l} \sum_{k}\left|c_{l k}\right|, \sup _{k} \sum_{l}\left|c_{l k}\right|\right\}\right)^{p} \sum_{n}\left|a_{n}\right|^{p},
$$

with the obvious meaning for $p=\infty$, where $c_{l k}, a_{l}$ are sequences of real or complex numbers. Indeed, for $p=1$ and $p=\infty$, (4.42) is easy to obtain. Then we obtain (4.42) for all $1 \leq p \leq \infty$ by interpolation. Let $z \in \mathbb{C}_{+}$and set $r^{2}=4\left(\operatorname{Re} z^{-1}\right)^{-1}$, that is, $r=2 \frac{|z|}{\sqrt{\operatorname{Re} z}}$. Let $x_{k}$ be a maximal sequence in $M$ such that all the balls $B\left(x_{k}, r / 2\right)$ are disjoint. Note that the balls $B\left(x_{k}, r\right)$ are such that $\cup_{k} B\left(x_{k}, r\right)=M$, and that by doubling there exists $N \in \mathbb{N}^{*}$ such that any $x \in M$ is contained in at most $N$ such balls. Let $\chi_{k}$ be the characteristic function of the set $B_{k}=B\left(x_{k}, r\right) \backslash \cup_{i=1}^{k-1} B\left(x_{i}, r\right)$, and set $r_{l k}=d\left(B_{k}, B_{l}\right)$. Using Jensen and doubling, we may write

$$
\begin{aligned}
\|\exp (-z L) f\|_{p}^{p} & \leq \sum_{k}\left(\sum_{l}\left\|\chi_{k} \exp (-z L)\left(\chi_{l} f\right)\right\|_{p}\right)^{p} \\
& \leq C \sum_{k}\left(\sum_{l} V\left(x_{k}, r\right)^{\frac{1}{p}-\frac{1}{2}}\left\|\mathbf{m}_{\chi_{k}} \exp (-z L) \mathbf{m}_{\chi_{l}}\right\|_{p \rightarrow 2}\left\|\chi_{l} f\right\|_{p}\right)^{p},
\end{aligned}
$$

hence by (4.42)

where

$$
\|\exp (-z L) f\|_{p}^{p} \leq C \max \{I, I I\}^{p}\|f\|_{p}^{p}
$$

$$
\begin{aligned}
I & =\sup _{l} \sum_{k} V\left(x_{k}, r\right)^{\frac{1}{p}-\frac{1}{2}}\left\|\mathbf{m}_{\chi_{k}} \exp (-z L) \mathbf{m}_{\chi_{l}}\right\|_{p \rightarrow 2}, \\
I I & =\sup _{k} \sum_{l} V\left(x_{k}, r\right)^{\frac{1}{p}-\frac{1}{2}}\left\|\mathbf{m}_{\chi_{k}} \exp (-z L) \mathbf{m}_{\chi_{l}}\right\|_{p \rightarrow 2} .
\end{aligned}
$$

Thus $\|\exp (-z L) f\|_{p \rightarrow p} \leq C \max \{I, I I\}$, and our goal is to estimate from above $I$ and $I I$ by $C\left(\frac{|z|}{\operatorname{Re} z}\right)^{\delta\left|\frac{1}{p}-\frac{1}{2}\right|}$; the result for the other values of $\tilde{p}$ follows by duality and interpolation. We shall explain how one deals with $I$, the treatment of $I I$ being similar. Write

$$
\sup _{l} \sum_{k} V\left(x_{k}, r\right)^{\frac{1}{p}-\frac{1}{2}}\left\|\mathbf{m}_{\chi_{k}} \exp (-z L) \mathbf{m}_{\chi_{l}}\right\|_{p \rightarrow 2}=I I I+I V,
$$


with

$$
I I I=\sup _{l} \sum_{\left\{k ; r_{k l} \leq 2 r\right\}} V\left(x_{k}, r\right)^{\frac{1}{p}-\frac{1}{2}}\left\|\mathbf{m}_{\chi_{k}} \exp (-z L) \mathbf{m}_{\chi_{l}}\right\|_{p \rightarrow 2}
$$

and

$$
I V=\sup _{l} \sum_{i=1}^{\infty} \sum_{\left\{k ; 2 i r<r_{k l} \leq 2(i+1) r\right\}} V\left(x_{k}, r\right)^{\frac{1}{p}-\frac{1}{2}}\left\|\mathbf{m}_{\chi_{k}} \exp (-z L) \mathbf{m}_{\chi_{l}}\right\|_{p \rightarrow 2}
$$

Observe first that there exists $C$ only depending on the doubling constant so that, for all $l, \#\left\{k ; r_{k l} \leq r\right\} \leq C$.

Therefore

$$
I I I \leq C \sup _{\left\{k, l ; r_{k l} \leq 2 r\right\}} V\left(x_{k}, r\right)^{\frac{1}{p}-\frac{1}{2}}\left\|\mathbf{m}_{\chi_{k}} \exp (-z L) \mathbf{m}_{\chi_{l}}\right\|_{p \rightarrow 2}
$$

The conclusion of the corollary is trivial for $p=2$, therefore we can assume $1 \leq p<2$. Using the contractivity of $\exp (-i s L)$ on $L^{2}(M, d \mu)$, one sees that (4.41) implies (4.36), with $\Psi(z)=\exp (-z L), q=2, W_{1} \equiv 1$ and $W_{2}=V^{(1 / p)-(1 / 2)}$.

Thus

$$
\begin{aligned}
\left\|\mathbf{m}_{\chi_{k}} \exp (-z L) \mathbf{m}_{\chi_{l}}\right\|_{p \rightarrow 2} & \leq\left\|\exp (-z L) \mathbf{m}_{\chi_{l}}\right\|_{p \rightarrow 2} \\
& \leq\left\|\exp (-z L) \mathbf{m}_{V^{(1 / p)-(1 / 2)}(., \sqrt{\operatorname{Re} z})}\right\|_{p \rightarrow 2}\left\|\mathbf{m}_{V^{(1 / 2)-(1 / p)}(., \sqrt{\operatorname{Re} z})} \mathbf{m}_{\chi_{l}}\right\|_{p \rightarrow p} \\
& \leq \sup _{x \in B_{l}} V^{\frac{1}{2}-\frac{1}{p}}(x, \sqrt{\operatorname{Re} z}) .
\end{aligned}
$$

Hence, if $r_{k l} \leq 2 r$,

$$
\begin{aligned}
I I I & \leq C \sup _{\left\{k, l ; r_{k l} \leq 2 r\right\}} V\left(x_{k}, r\right)^{\frac{1}{p}-\frac{1}{2}}\left\|\mathbf{m}_{\chi_{k}} \exp (-z L) \mathbf{m}_{\chi_{l}}\right\|_{p \rightarrow 2} \\
& \leq C \sup _{\left\{k, l ; r_{k l} \leq 2 r\right\}} \sup _{x \in B_{l}}\left(\frac{V\left(x_{k}, r\right)}{V(x, \sqrt{\operatorname{Re} z})}\right)^{\frac{1}{p}-\frac{1}{2}} \\
& \leq C \sup _{\left\{k, l ; r_{k l} \leq 2 r\right\}} \sup _{x \in B_{l}}\left(\frac{V\left(x, 4 r+r_{k l}\right)}{V(x, \sqrt{\operatorname{Re} z})}\right)^{\frac{1}{p}-\frac{1}{2}} \\
& \leq C\left(\frac{V(x, 6 r)}{V(x, \sqrt{\operatorname{Re} z})}\right)^{\frac{1}{p}-\frac{1}{2}} \\
& \leq C(r / \sqrt{\operatorname{Re} z})^{\delta\left(\frac{1}{p}-\frac{1}{2}\right)} \\
& \leq C(|z| / \operatorname{Re} z)^{\delta\left(\frac{1}{p}-\frac{1}{2}\right)} .
\end{aligned}
$$

In the last inequality, we could use doubling since $r=2 \frac{|z|}{\sqrt{\operatorname{Re} z}} \geq \sqrt{\operatorname{Re} z}$.

Now for $I V$. Again, there exists $C$ only depending on the doubling constant so that, for all $l, \#\left\{k ; r_{k l} \leq i r\right\} \leq C i^{\delta}$.

Therefore

$$
I V \leq C \sup _{l} \sum_{i=1}^{\infty} i^{\delta} \sup _{\left\{k ; 2 i r<r_{k l} \leq 2(i+1) r\right\}} V^{\frac{1}{p}-\frac{1}{2}}\left(x_{k}, r\right)\left\|\mathbf{m}_{\chi_{k}} \exp (-z L) \mathbf{m}_{\chi_{l}}\right\|_{p \rightarrow 2}
$$

Let us now estimate $\left\|\mathbf{m}_{\chi_{k}} \exp (-z L) \mathbf{m}_{\chi_{l}}\right\|_{p \rightarrow 2}$ for $r_{k l}>2 r$ with the help of Theorem 4.13. Note that $\delta_{1}=0$ and $\delta_{2}=\delta\left(\frac{1}{p}-\frac{1}{2}\right)$, where $\delta$ is the exponent in (4.10). 
With our choice of $r, z \in \mathcal{C}_{r_{k l}^{2} / 4}$ as soon as $r_{k l} \geq r$. Therefore (4.37) yields

$$
\begin{aligned}
& \left\|\mathbf{m}_{\chi_{k}} \exp (-z L) \mathbf{m}_{\chi_{l}}\right\|_{p \rightarrow 2} \\
\leq & \left\|\mathbf{m}_{\chi_{k}} \exp (-z L) \mathbf{m}_{V^{(1 / p)-(1 / 2)}\left(., r_{k l} / 2\right)} \mathbf{m}_{\chi_{l}}\right\|_{p \rightarrow 2}\left\|\mathbf{m}_{V^{(1 / 2)-(1 / p)}\left(., r_{k l} / 2\right)} \mathbf{m}_{\chi_{l}}\right\|_{p \rightarrow p} \\
\leq & C\left(\frac{r_{k l}^{2}}{4|z|}\right)^{\delta\left(\frac{1}{p}-\frac{1}{2}\right)} \exp \left(-\operatorname{Re} \frac{r_{k l}^{2}}{4 z}\right)\left\|\mathbf{m}_{V^{(1 / 2)-(1 / p)}\left(., r_{k l} / 2\right)} \mathbf{m}_{\chi_{l}}\right\|_{p \rightarrow p} .
\end{aligned}
$$

Now

$$
\begin{aligned}
& \left\|\mathbf{m}_{V^{(1 / 2)-(1 / p)\left(., r_{k l} / 2\right)}} \mathbf{m}_{\chi_{l}}\right\|_{p \rightarrow p} \leq \sup _{x \in B_{l}} V^{\frac{1}{2}-\frac{1}{p}}\left(x, r_{k l} / 2\right) \\
& \leq\left(\inf _{x \in B_{l}} V\left(x, r_{k l} / 2\right)\right)^{\frac{1}{2}-\frac{1}{p}} \\
& \leq V^{\frac{1}{p}-\frac{1}{2}}\left(x_{l},\left(r_{k l} / 2\right)-r\right) \\
& \leq V^{\frac{1}{p}-\frac{1}{2}}\left(x_{l},(i-1) r\right) \text {. }
\end{aligned}
$$

Thus, if $2 i r<r_{k l} \leq 2(i+1) r$,

$$
\begin{aligned}
\left\|\mathbf{m}_{\chi_{k}} \exp (-z L) \mathbf{m}_{\chi_{l}}\right\|_{p \rightarrow 2} & \leq\left(\frac{r_{k l}^{2}}{4|z|}\right)^{\delta\left(\frac{1}{p}-\frac{1}{2}\right)} \exp \left(-\operatorname{Re} \frac{r_{k l}^{2}}{4 z}\right) V^{\frac{1}{2}-\frac{1}{p}}\left(x_{l},(i-1) r\right) \\
& \leq V^{\frac{1}{2}-\frac{1}{p}}\left(x_{l},(i-1) r\right)\left(\frac{(i+1)^{2} r^{2}}{|z|}\right)^{\delta\left(\frac{1}{p}-\frac{1}{2}\right)} \exp \left(-i^{2} r^{2} \operatorname{Re} \frac{1}{z}\right) \\
& =V^{\frac{1}{2}-\frac{1}{p}}\left(x_{l},(i-1) r\right)\left(\frac{(i+1)^{2} r^{2}}{|z|}\right)^{\delta\left(\frac{1}{p}-\frac{1}{2}\right)} e^{-4 i^{2}} .
\end{aligned}
$$

Finally,

$$
I V \leq C \sup _{l} \sum_{i=2}^{\infty} i^{\delta} \sup _{2 i r<r_{k l} \leq 2(i+1) r}\left(\frac{V\left(x_{k}, r\right)}{V\left(x_{l},(i-1) r\right)}\right)^{\frac{1}{p}-\frac{1}{2}}\left(\frac{(i+1)^{2} r^{2}}{|z|}\right)^{\delta\left(\frac{1}{p}-\frac{1}{2}\right)} e^{-4 i^{2}} .
$$

By doubling,

$$
\left(\frac{V\left(x_{k}, r\right)}{V\left(x_{l},(i-1) r\right)}\right)^{\frac{1}{p}-\frac{1}{2}} \leq\left(\frac{V\left(x_{l}, 3 r+r_{k l}\right)}{V\left(x_{l},(i-1) r\right)}\right)^{\frac{1}{p}-\frac{1}{2}} \leq\left(\frac{V\left(x_{l},(2 i+5) r\right)}{V\left(x_{l},(i-1) r\right)}\right)^{\frac{1}{p}-\frac{1}{2}}
$$

is uniformly bounded. Therefore

$$
I V \leq C \sum_{i=2}^{\infty} i^{\delta}\left(\frac{(i+1)^{2} r^{2}}{|z|}\right)^{\delta\left(\frac{1}{p}-\frac{1}{2}\right)} e^{-4 i^{2}} \leq C\left(\sum_{i=2}^{\infty} e^{-4 i^{2} /(1+\varepsilon)}\right)(|z| / \operatorname{Re} z)^{\delta\left(\frac{1}{p}-\frac{1}{2}\right)}
$$

which finishes the proof.

We finish this section with the description of a simple and natural example of a family of operators which generate semigroups without heat kernel. We consider the following family of self-adjoint operators

$$
L^{(c)}=\Delta-c|x|^{-2}
$$

acting on $L^{2}\left(\mathbb{R}^{n}\right)$ for $n \geq 3$, where $\Delta=-\sum_{i=1}^{n} \partial_{x_{i}}^{2}$ and $0, c \leq(n-2)^{2} / 4$. Hardy's inequality shows that

$$
\Delta \geq(n-2)^{2} / 4|x|^{2}
$$


(see for example [55, (2.1), p.107]). Hence, for all $c \in\left[0,(n-2)^{2} / 4\right], L^{(c)}$ is non-negative. A detailed discussion of the definition of the operators $L^{(c)}$ can be found for example in 3 . Such operators are called Schrödinger operators with the inverse-square potential and they are of substantial interest in analysis (see for example [13, 55] and references therein). Note that $L^{(c)}$ is homogeneous of order 2, meaning that if $U_{t}$ is the dilation $\left(U_{t} f\right)(x)=f(t x)$, $t>0, x \in \mathbb{R}^{n}$, then $U_{1 / t} L^{(c)} U_{t}=t^{2} L^{(c)}$ for all $t>0$. As a consequence,

$$
\left\|\exp \left(-t L^{(c)}\right)\right\|_{q \rightarrow p}=t^{\frac{n}{2}\left(\frac{1}{q}-\frac{1}{p}\right)}\left\|\exp \left(-L^{(c)}\right)\right\|_{q \rightarrow p}
$$

Set

$$
C_{c, p, q}=\left\|\exp \left(-L^{(c)}\right)\right\|_{p \rightarrow q} .
$$

It was proved in [55. Corollary 6.2] that $C_{c, p, q}=\infty$ for all $p \leq q$ and $q>p_{c}^{*}=n / \sigma$, where $\sigma=(n-2) / 2-\sqrt{(n-2)^{2} / 4-c}$. It means in particular that for every $t>0$ the operator $\exp \left(-t L^{(c)}\right)$ cannot be extended to a bounded operator on $L^{p}\left(\mathbb{R}^{n}\right)$ for $p>p *_{c}$, hence $L^{(c)}$ does not generate a semigroup on $L^{p}\left(\mathbb{R}^{n}\right)$ for such $p$. It also means that for every $t>0$ the operator $\exp \left(-t L^{(c)}\right)$ cannot be extended to a bounded operator from $L^{1}\left(\mathbb{R}^{n}\right)$ to $L^{\infty}\left(\mathbb{R}^{n}\right)$ : its kernel is not a bounded function on $\mathbb{R}^{2 n}$ but merely a distribution. Therefore standard heat kernel theory can not be applied to study the semigroup generated by $L^{(c)}$. However, it was proved in [56], see also [42], that $\left\|\exp \left(-L^{(c)}\right)\right\|_{p \rightarrow p}$ is finite for all $p$ in the interval $\left(\left(p_{c}^{*}\right)^{\prime}, p_{c}^{*}\right)$.

Our main interest here is some form of Gaussian type estimates which we can obtain for $L^{(c)}$ even though we know that that the pointwise Gaussian estimates cannot hold. Our $L^{p} \rightarrow L^{q}$ Gaussian estimates are described in the following example.

Example 4.15. Suppose that $c<(n-2)^{2} / 4$ and that $p_{*}^{\prime}<p<2<q<p_{*}$, where $1 / p_{*}+1 / p_{*}^{\prime}=1, p_{*}=n / \sigma_{c}$ and $\sigma_{c}=(n-2) / 2-\sqrt{(n-2)^{2} / 4-c}$. Then there exists a constant $C$ such that for any two open subsets of $U_{1}, U_{2} \subset \mathbb{R}^{n}$ the following estimates hold

$$
\left\|P_{1} \exp \left(-z L^{(c)}\right) P_{2}\right\|_{p \rightarrow q} \leq C(\operatorname{Re} z)^{-\nu}\left(1+\operatorname{Re} \frac{r^{2}}{4 z}\right)^{\nu} \exp \left(-\operatorname{Re} \frac{r^{2}}{4 z}\right), \forall z \in \mathbb{C}_{+},
$$

where $r=d\left(U_{1}, U_{2}\right), d$ is the Euclidean distance, $P_{i}=\mathbf{m}_{\chi_{U_{i}}}$ and $\nu=\frac{n}{2}\left(\frac{1}{p}-\frac{1}{q}\right)$.

Proof. By Theorem 3.3, $L^{(c)}$ satisfies Davies-Gaffney condition (3.2), thus finite propagation speed for the corresponding wave equation with the standard Euclidean distance. We need

$$
\left\|\exp \left(-t L^{(c)}\right)\right\|_{2 \rightarrow q} \leq C t^{\frac{n}{2}\left(\frac{1}{q}-\frac{1}{2}\right)}, \quad \forall t \in \mathbb{R}_{+},
$$

for all $p$ such that $2 \leq p \leq p_{*}$. This is proved in [27, Theorem 11 and Lemma 13], but for the bounded potential $1 /\left(1+|x|^{2}\right)$ instead of $1 /|x|^{2}$. To circumvent this, we proceed as in the proof of Theorem 3.3. setting $L_{a}^{(c)}=-\Delta+\mathcal{V}_{a}$, where $\mathcal{V}_{a}=\max \left\{-c|x|^{-2},-a\right\}$, and we notice that, when $a$ goes to $\infty, L_{a}$ converges to $L+\mathcal{V}$ in the strong resolvent sense (see 40 , Theorem VIII.3.3, p.454] or [48, Theorem S.16 p.373]). This yields (4.43). Moreover,

$$
\left\|\exp \left((-t+i s) L^{(c)}\right)\right\|_{p \rightarrow q} \leq\left\|\exp \left(-t L^{(c)} / 2\right)\right\|_{2 \rightarrow q}\left\|\exp \left(-t L^{(c)} / 2\right)\right\|_{p \rightarrow 2}
$$

So

$$
\left\|\exp \left(-z L^{(c)}\right)\right\|_{p \rightarrow q} \leq C(\operatorname{Re} z)^{\frac{n}{2}\left(\frac{1}{q}-\frac{1}{p}\right)}
$$

for all $z \in \mathbb{C}_{+}$and all $p, q$ such that $p_{*}^{\prime}<p \leq q<p_{*}$. 
Hence for $p, q$ such that $p_{*}^{\prime}<q \leq p<p_{*}, L^{(c)}$ satisfies all assumptions of Theorem 4.13 with $W_{1}(x, t)=C t^{\frac{n}{2}\left(\frac{1}{2}-\frac{1}{q}\right)}$ and $W_{2}(x, t)=C t^{\frac{n}{2}\left(\frac{1}{p}-\frac{1}{2}\right)}, K_{1}^{\prime}=K_{2}^{\prime}=1, \delta_{1}=\frac{n}{2}\left(\frac{1}{2}-\frac{1}{q}\right)$ and $\delta_{2}=\frac{n}{2}\left(\frac{1}{p}-\frac{1}{2}\right)$. Thus Theorem 4.13 yields

$$
\begin{aligned}
\left\|P_{1} \exp \left(-z L^{(c)}\right) P_{2}\right\|_{p \rightarrow q} & \leq C r^{-\left(\delta_{1}+\delta_{2}\right)}\left|\frac{r^{2}}{4 z}\right|^{\delta_{1}+\delta_{2}} \exp \left(-\operatorname{Re} \frac{r^{2}}{4 z}\right) \\
& =C(\operatorname{Re} z)^{-\left(\delta_{1}+\delta_{2}\right)}\left(\operatorname{Re} \frac{r^{2}}{4 z}\right)^{\left(\delta_{1}+\delta_{2}\right) / 2} \exp \left(-\operatorname{Re} \frac{r^{2}}{4 z}\right)
\end{aligned}
$$

for all $z \in \mathcal{C}_{r^{2} / 4}$. The estimate for $z \notin \mathcal{C}_{r^{2} / 4}$ follows directly from (4.44).

4.7. Other functional spaces. Our approach allows us to state and prove an analog of offdiagonal bounds for other functional spaces. Suppose that $B$ is a Banach space of functions on $M$ and that $L^{2}(M, d \mu) \cap B$ is dense in $B$ with respect to its norm. Suppose next that $U$ is an open subset of $M$. We define the space $B(U)$ as the closure of $L^{2}(U, d \mu) \cap B$ in the space $B$. Then we define $B^{*}(U)$ as the space $B^{*}$ with seminorm given by the formula

$$
\|f\|_{B^{*}(U)}=\sup _{\|g\|_{B(U)} \leq 1}\langle f, g\rangle,
$$

where $\langle f, g\rangle$ is the duality pairing.

Theorem 4.16. Let $(M, d, \mu)$ be a metric measure space, $B_{1}$ and $B_{2}$ Banach spaces as above, and $U_{1}, U_{2}$ open subsets of $M$. Set $r=d\left(U_{1}, U_{2}\right)$. Let $g: \mathbb{C}_{+} \rightarrow \mathbb{C}$ be an analytic function which satisfies condition (2.14) for $\gamma=\frac{r^{2}}{4}$, and let $\left\{\Psi(z): z \in \mathbb{C}_{+}\right\}$be a family of bounded linear operators on $L^{2}(M, d \mu)$ satisfying conditions (3.1), (3.2). Assume that

$$
\|\Psi(z)\|_{B_{1} \rightarrow B_{2}^{*}} \leq(\operatorname{Re} z)^{-D / 2} \exp (-\operatorname{Re} g(z)), \forall z \in \mathbb{C}_{+} .
$$

Then, for all $z \in \mathbb{C}_{+}$,

$$
\|\Psi(z)\|_{B_{1}\left(U_{1}\right) \rightarrow B_{2}^{*}\left(U_{2}\right)} \leq(\operatorname{Re} z)^{-D / 2}\left(1+\operatorname{Re} \frac{r^{2}}{4 z}\right)^{D / 2} \exp \left(1-\operatorname{Re} g(z)-\operatorname{Re} \frac{r^{2}}{4 z}\right) .
$$

About the growth condition (2.14), the same remark is in order than after Theorem 4.6. Proof. For $f_{i} \in L^{2}\left(U_{i}, d \mu\right) \cap B_{i}, i=1,2$, we again consider the function $F$ defined by the formula

By assumption

$$
F(z)=\left\langle\Psi(z) f_{2}, f_{1}\right\rangle
$$

$$
\begin{gathered}
|F(z)| \leq(\operatorname{Re} z)^{-D / 2} \exp (-\operatorname{Re} g(z))\left\|f_{1}\right\|_{B_{1}\left(U_{1}\right)}\left\|f_{2}\right\|_{B_{2}\left(U_{2}\right)}, \quad z \in \mathbb{C}_{+}, \\
|F(z)| \leq\left\|f_{1}\right\|_{L^{2}\left(U_{1}, d \mu\right)}\left\|f_{2}\right\|_{L^{2}\left(U_{2}, d \mu\right)}, \quad z \in \mathbb{C}_{+},
\end{gathered}
$$

and

$$
|F(t)| \leq \exp \left(-\frac{r^{2}}{4 t}\right)\left\|f_{1}\right\|_{L^{2}\left(U_{1}, d \mu\right)}\left\|f_{2}\right\|_{L^{2}\left(U_{2}, d \mu\right)}, \quad t>0 .
$$

Therefore Proposition 2.4 yields

$$
|F(z)| \leq(\operatorname{Re} z)^{-D / 2}\left(1+\operatorname{Re} \frac{r^{2}}{4 z}\right)^{D / 2} \exp \left(-\operatorname{Re} g(z)-\operatorname{Re} \frac{r^{2}}{4 z}\right)\left\|f_{1}\right\|_{B_{1}\left(U_{1}\right)}\left\|f_{2}\right\|_{B_{2}\left(U_{2}\right)}
$$

hence the claim. 
Example 4.17. Let $\Delta$ be the Laplace-Beltrami operator acting on a complete Riemannian manifold $M$. Assume that, for some $p \in(1, \infty)$ and some $\alpha \geq 0$,

$$
\|\exp (-z \Delta)\|_{p \rightarrow p} \leq C\left(\frac{|z|}{\operatorname{Re} z}\right)^{\alpha}, \forall z \in \mathbb{C}_{+} .
$$

Then, for any pair $U_{1}, U_{2}$ of open subsets of $M$, one has

$$
\left(\int_{U_{2}}|\exp (-z \Delta) f|^{p} d \mu\right)^{1 / p} \leq C\left(\frac{|z|}{\operatorname{Re} z}\right)^{\alpha}\left(1+\operatorname{Re} \frac{r^{2}}{4 z}\right)^{\alpha} \exp \left(-\operatorname{Re} \frac{r^{2}}{4 z}\right)\|f\|_{L^{p}\left(U_{1}, d \mu\right)}^{2},
$$

where $r=d\left(U_{1}, U_{2}\right)$, for all $z \in \mathbb{C}_{+}$and $f \in L^{p}\left(U_{1}, d \mu\right)$.

Proof. Note that in virtue of our assumptions the semigroup $\exp (-z \Delta), z \in \mathbb{C}_{+}$satisfies condition (4.45) with $B_{1}=L^{p}(M), B_{2}=L^{p^{\prime}}(M), D=2 \alpha$ and $\left|e^{g(z)}\right|=|z|^{\alpha}$. Hence Example 4.17 follows from Theorem 4.16 .

For the relevance of assumption (4.46), see [14], 9], and Corollary 4.14 above.

We can also set $g=\exp (\lambda z)$ in Theorem 4.16, For $\lambda>0$, this allows one to treat the case where one only has small time on-diagonal upper bounds, for $\lambda>0$, this allows one, in case there is a spectral gap in the on-diagonal bounds, to keep track of it in the off-diagonal ones (see [47] for more in this direction). Such modification can be made in all our previous statements leading from on-diagonal bounds to off-diagonal bounds. We leave the details to the reader.

4.8. Possible further generalizations. The technique presented above is very flexible. For instance, in the statement and the proof of Theorem 4.13 and many other results, we do not have to assume that the operators $\Psi(z)$ are linear. We think that it should be possible to find non-linear examples where the Phragmén-Lindelöf technique yields interesting results. Also, other pairs of dual norms than the $\left(L^{2}, L^{2}\right)$ norms could be considered in (3.1) and (3.2). Of course, in such cases, one would loose the connection with finite speed propagation.

4.9. Open question. Can one treat in a similar way the so-called sub-Gaussian estimates (see for instance [6], [17]), which are typical of fractals, namely, can one imagine that, in the above notation,

$$
\left|\left\langle e^{-t L} f_{1}, f_{2}\right\rangle\right| \leq \exp \left(-c\left(\frac{r^{\beta}}{t}\right)^{\frac{1}{\beta-1}}\right)\left\|f_{1}\right\|_{2}\left\|f_{2}\right\|_{2},
$$

where $r$ is the distance between the supports of $f_{1}$ and $f_{2}$, and

$$
p_{t}(x, x) \leq \frac{C}{V\left(x, t^{1 \beta}\right)},
$$

imply

for $\beta>2$ ?

$$
p_{t}(x, y) \leq \frac{C^{\prime}}{V\left(x, t^{1 / \beta}\right)} \exp \left(-c^{\prime}\left(\frac{d^{\beta}(x, y)}{t}\right)^{\frac{1}{\beta-1}}\right),
$$

Acknowledgements: The first-named author would like to thank several institutions where he could find the necessary peace of mind to work on this, in particular Macquarie 
University, Sydney, the University of Cyprus, the Isaac Newton Institute, Cambridge, and respectively Xuan-Thinh Duong, Georgios Alexopoulos, and the organizers of the program on Spectral theory and partial differential equations, for giving him the opportunity to stay there. The second-named author would like to thank Derek Robinson for organizing his extended visit to the Australian National University, where part of this work was carried out. Both authors would like to thank Brian Davies, Alexander Grigor'yan, Alexander Teplyaev, whose questions helped to improve the manuscript.

\section{REFERENCES}

[1] G. Alexopoulos. Spectral multipliers on Lie groups of polynomial growth. Proc. Amer. Math. Soc., 120(3):973-979, 1994.

[2] G. Alexopoulos. Oscillating multipliers on Lie groups and Riemannian manifolds. Tohoku Math. J. (2), 46(4):457-468, 1994.

[3] W. Arendt, G.R. Goldstein and J.A. Goldstein. Outgrowths of Hardy's inequality, preprint, 2006.

[4] P. Auscher, T. Coulhon, X.T. Duong and S. Hofmann. Riesz transform on manifolds and heat kernel regularity. Ann. Sc. E. N. S., 37:911-957, 2004.

[5] P. Auscher and J.-M. Martell. Weighted norm inequalities, off-diagonal estimates and elliptic operators, Part II: Off-diagonal estimates on spaces of homogeneous type, preprint, 2005.

[6] M. Barlow. Diffusions on fractals, in Lectures on probability theory and Statistics. Ecole d'été de probabilités de St-Flour, XXV, 1995, Springer Lecture Notes in Math., 1690, 1-121, 1998.

[7] A. Bendikov and L. Saloff-Coste. On- and off-diagonal heat kernel behaviors on certain infinite dimensional local Dirichlet spaces. American J. Math. , 122, 1205-1263, 2000.

[8] S. Blunck. A Hörmander-type spectral multiplier theorem for operators without heat kernel. Ann. Sc. Norm. Super. Pisa Cl. Sci. (5), 2(3):449-459, 2003.

[9] S. Blunck. Generalized Gaussian estimates and Riesz means of Schrödinger groups, unpublished manuscript, 2003.

[10] S. Blunck and P.C. Kunstmann. Calderón-Zygmund theory for non-integral operators and the $H^{\infty}$ functional calculus. Rev. Mat. Iberoamericana, 19(3):919-942, 2003.

[11] S. Blunck and P.C. Kunstmann. Weak type $(p, p)$ estimates for Riesz transforms. Math. Z., 247(1):137$148,2004$.

[12] S. Blunck and P.C. Kunstmann. Generalized Gaussian estimates and the Legendre transform. J. Operator Th., 53(2):351-365, 2005.

[13] N. Burq, F. Planchon, J.G. Stalker, and A.S. Tahvildar-Zadeh. Strichartz estimates for the wave and Schrödinger equations with the inverse-square potential. J. Funct. Anal., 203(2):519-549, 2003.

[14] G. Carron, T. Coulhon, and E.-M. Ouhabaz. Gaussian estimates and $L^{p}$-boundedness of Riesz means. J. Evol. Equ., 2(3):299-317, 2002.

[15] M. Christ. $L^{p}$ bounds for spectral multipliers on nilpotent groups. Trans. Amer. Math. Soc., 328(1):7381, 1991.

[16] T. Coulhon. Itération de Moser et estimation gaussienne du noyau de la chaleur. J. Operator Theory, 29(1):157-165, 1993.

[17] T. Coulhon. Off-diagonal heat kernel lower bounds without Poincaré. J. London Math. Soc., 68(3):795816, 2003.

[18] T. Coulhon and X.T. Duong. Riesz transforms for $1 \leq p \leq 2$. Trans. Amer. Math. Soc., 351(3):1151$1169,1999$.

[19] T. Coulhon and X. T. Duong. Maximal regularity and kernel bounds: observations on a theorem by Hieber and Prüss. Adv. Diff. Eq., 5(1-3):343-368, 2000.

[20] T. Coulhon and X.T. Duong. Riesz transforms for $p>$ 2. C. R. Acad. Sci. Paris Sér. I Math., 332(11):975-980, 2001.

[21] T. Coulhon and X.T. Duong. Riesz transform and related inequalities on noncompact Riemannian manifolds. Comm. Pure Appl. Math., 56(12):1728-1751, 2003.

[22] T. Coulhon, A. Grigor'yan and F. Zucca. The discrete integral maximum principle and its applications. Tohoku Math. J., 57(4):559-587, 2005. 
[23] E. B. Davies. Heat kernels and spectral theory. Cambridge University Press, Cambridge, 1989.

[24] E. B. Davies. Heat kernel bounds, conservation of probability and the Feller property. J. Anal. Math., 58:99-119, 1992. Festschrift on the occasion of the 70th birthday of Shmuel Agmon.

[25] E. B. Davies. Uniformly elliptic operators with measurable coefficients. J. Funct. Anal., 132(1):141-169, 1995.

[26] E. B. Davies and M. M. H. Pang. Sharp heat kernel bounds for some Laplace operators. Quart. J. Math. Oxford Ser. (2), 40(159):281-290, 1989.

[27] E. B. Davies and B. Simon. $L^{p}$ norms of noncritical Schrödinger semigroups. J. Funct. Anal., 102(1):95$115,1991$.

[28] X. T. Duong, E. M. Ouhabaz, and A. Sikora. Plancherel-type estimates and sharp spectral multipliers. J. Funct. Anal., 196(2):443-485, 2002.

[29] N. Dunford and J. T. Schwartz. Linear operators. Part I. Wiley Classics Library. John Wiley \& Sons Inc., New York, 1988. General theory, with the assistance of William G. Bade and Robert G. Bartle, reprint of the 1958 original, a Wiley-Interscience publication.

[30] N. Dungey. Some remarks on gradient estimates for heat kernels. Abstr. Appl. Anal., 73020, 2006.

[31] M. P. Gaffney. The conservation property of the heat equation on Riemannian manifolds. Comm. Pure Appl. Math., 12:1-11, 1959.

[32] J. Galé and T. Pytlik. Functional calculus for infinitesimal generators of holomorphic semigroups. $J$. Funct. Anal., 150(2): 307-355, 1997.

[33] A. Grigor'yan. Heat kernel upper bounds on a complete non-compact manifold. Rev. Mat. Iberoamericana, 10(2):395-452, 1994.

[34] A. Grigor'yan. Gaussian upper bounds for the heat kernel on arbitrary manifolds. J. Diff. Geom., 45(1):33-52, 1997.

[35] A. Grigor'yan. Estimates of heat kernels on Riemannian manifolds, in Spectral theory and geometry (Edinburgh, 1998), London Math. Soc. Lecture Note Ser., 273, 140-225. Cambridge Univ. Press, Cambridge, 1999.

[36] A. Grigor'yan. Heat kernel upper bounds on fractal spaces, preprint, 2004.

[37] W. Hebisch. Almost everywhere summability of eigenfunction expansions associated to elliptic operators. Studia Math., 96(3):263-275, 1990.

[38] M. Hino and J. Ramirez. Small-time Gaussian behavior of symmetric diffusion semigroups. Ann. Probab., 31(3):1254-1295, 2003.

[39] L. Hörmander. The analysis of linear partial differential operators. I. Distribution theory and Fourier analysis. Second edition. Grundlehren der Mathematischen Wissenschaften 256. Springer-Verlag, Berlin, 1990.

[40] T. Kato. Perturbation theory for linear operators. Classics in Mathematics. Springer-Verlag, Berlin, 1995. Reprint of the 1980 edition.

[41] M. Keel and T. Tao. Endpoint Strichartz estimates. Amer. J. Math., 120(5):955-980, 1998.

[42] V. Liskevich, Z. Sobol, H. Vogt. On $L_{p}$-theory of $C_{0}$-semigroups associated with second order elliptic operators. J. Funct. Anal., 193:55-76, 2002.

[43] M. Marias, E. Russ. $H^{1}$-boundedness of Riesz transforms and imaginary powers of the Laplacian on Riemannian manifolds. Ark. Mat., 41(1):115-132, 2003.

[44] A. I. Markushevich. Theory of functions of a complex variable. Vol. I, II, III. Chelsea Publishing Co., New York, English edition, 1977. Translated and edited by Richard A. Silverman.

[45] S. A. Molchanov. Diffusion processes, and Riemannian geometry. Uspehi Mat. Nauk, 30(1(181)):3-59, 1975.

[46] E. M. Ouhabaz. Analysis of heat equations on domains. London Mathematical Society Monographs Series, 31. Princeton University Press, Princeton, NJ, 2005.

[47] E. M. Ouhabaz. Comportement des noyaux de la chaleur des opérateurs de Schrödinger et applications à certaines équations paraboliques semi-linéaires, to appear in J. Funct. Anal. .

[48] M. Reed and B. Simon. Methods of modern mathematical physics. I. Functional analysis. Academic Press Inc. [Harcourt Brace Jovanovich Publishers], New York, second edition, 1980.

[49] A. Sikora. Sharp pointwise estimates on heat kernels. Quart. J. Math. Oxford Ser. (2), 47(187):371-382, 1996. 
[50] A. Sikora. On-diagonal estimates on Schrödinger semigroup kernels and reduced heat kernels. Comm. Math. Phys., 188(1):233-249, 1997.

[51] A. Sikora. Riesz transform, Gaussian bounds and the method of wave equation. Math. Z., 247(3):643$662,2004$.

[52] E. M. Stein and G. Weiss. Introduction to Fourier analysis on Euclidean spaces. Princeton University Press, Princeton, N.J., 1971. Princeton Mathematical Series, No. 32.

[53] K.-Th. Sturm. Analysis on local Dirichlet spaces. II. Upper Gaussian estimates for the fundamental solutions of parabolic equations. Osaka J. Math., 32(2):275-312, 1995.

[54] K.-Th. Sturm. The geometric aspect of Dirichlet forms. In New directions in Dirichlet forms, volume 8 of AMS/IP Stud. Adv. Math., pages 233-277. Amer. Math. Soc., Providence, RI, 1998.

[55] J. L. Vazquez and E. Zuazua. The Hardy inequality and the asymptotic behaviour of the heat equation with an inverse-square potential. J. Funct. Anal., 173(1):103-153, 2000.

[56] H. Vogt. $L_{p}$-properties of second order elliptic differential equations, $P h D$ Thesis, Dresden 2001.

[57] V.V. Zhikov. Spectral approach to asymptotic problems in diffusion. Diff. Equations, 25:33-39, 1989.

Thierry Coulhon, Département de Mathématiques, Université de Cergy-Pontoise, Site de Saint-Martin, 2, rue Adolphe Chauvin, F 95302 Cergy-Pontoise Cedex, FRAnCE

E-mail address: Thierry.Coulhon@math.u-cergy.fr

Adam Sikora, Department of Mathematical Sciences, New Mexico State University, Las Cruces, NM 88003-8001, USA

E-mail address: asikora@nmsu.edu 